\title{
A System of Mixed Equilibrium Problems, a General System of Variational Inequality Problems for Relaxed Cocoercive, and Fixed Point Problems for Nonexpansive Semigroup and Strictly Pseudocontractive Mappings
}

\author{
Poom Kumam ${ }^{1,2}$ and Phayap Katchang, ${ }^{2,3}$ \\ ${ }^{1}$ Department of Mathematics, Faculty of Science, King Mongkut's University of Technology Thonburi \\ (KMUTT), Bangkok 10140, Thailand \\ ${ }^{2}$ Centre of Excellence in Mathematics, CHE, Si Ayutthaya Road, Bangkok 10400, Thailand \\ ${ }^{3}$ Department of Mathematics and Statistics, Faculty of Science and Agricultural Technology, \\ Rajamangala University of Technology Lanna Tak, Tak 63000, Thailand
}

Correspondence should be addressed to Phayap Katchang, p.katchang@hotmail.com

Received 17 November 2011; Accepted 23 January 2012

Academic Editor: Giuseppe Marino

Copyright ( 2012 P. Kumam and P. Katchang. This is an open access article distributed under the Creative Commons Attribution License, which permits unrestricted use, distribution, and reproduction in any medium, provided the original work is properly cited.

We introduce an iterative algorithm for finding a common element of the set of solutions of a system of mixed equilibrium problems, the set of solutions of a general system of variational inequalities for Lipschitz continuous and relaxed cocoercive mappings, the set of common fixed points for nonexpansive semigroups, and the set of common fixed points for an infinite family of strictly pseudocontractive mappings in Hilbert spaces. Furthermore, we prove a strong convergence theorem of the iterative sequence generated by the proposed iterative algorithm under some suitable conditions which solves some optimization problems. Our results extend and improve the recent results of Chang et al. (2010) and many others.

\section{Introduction}

Let $H$ be a real Hilbert space with inner product $\langle\cdot, \cdot\rangle$ and norm $\|\cdot\|$. Let $C$ be a nonempty closed convex subset of $H$. Recall that a mapping $T: C \rightarrow C$ is nonexpansive if

$$
\|T x-T y\| \leq\|x-y\|, \quad \forall x, y \in C .
$$


We denote the set of fixed points of $T$ by $F(T)$, that is $F(T)=\{x \in C: x=T x\}$. A mapping $f: C \rightarrow C$ is said to be an $\alpha$-contraction if there exists a coefficient $\alpha \in(0,1)$ such that

$$
\|f(x)-f(y)\| \leq \alpha\|x-y\|, \quad \forall x, y \in C
$$

Let $B: C \rightarrow H$ be a mapping. Then $B$ is called:

(1) monotone if

$$
\langle B x-B y, x-y\rangle \geq 0, \quad \forall x, y \in C
$$

(2) $d$-strongly monotone if there exists a positive real number $d$ such that

$$
\langle B x-B y, x-y\rangle \geq d\|x-y\|^{2}, \quad \forall x, y \in C,
$$

for constant $d>0$, this implies that

$$
\|B x-B y\| \geq d\|x-y\|,
$$

that is, $B$ is $d$-expansive and when $d=1$, it is expansive;

(3) L-Lipschitz continuous if there exists a positive real number $L$ such that

$$
\|B x-B y\| \leq L\|x-y\|, \quad \forall x, y \in C ;
$$

(4) $c$-cocoercive $[1,2]$ if there exists a positive real number $c$ such that

$$
\langle B x-B y, x-y\rangle \geq c\|B x-B y\|^{2}, \quad \forall x, y \in C,
$$

Clearly, every $c$-cocoercive map $B$ is $(1 / c)$-Lipschitz continuous;

(5) relaxed c-cocoercive, if there exists a positive real number $c$ such that

$$
\langle B x-B y, x-y\rangle \geq(-c)\|B x-B y\|^{2}, \quad \forall x, y \in C
$$

(6) relaxed $(c, d)$-cocoercive, if there exists a positive real number $c, d$ such that

$$
\langle B x-B y, x-y\rangle \geq(-c)\|B x-B y\|^{2}+d\|x-y\|^{2}, \quad \forall x, y \in C,
$$

for $c=0, B$ is $d$-strongly monotone. This class of mapping is more general than the class of strongly monotone mapping. It is easy to see that we have the following implication: $d$-strongly monotonicity implying relaxed $(c, d)$-cocoercivity, 
(7) $k$-strictly pseudocontractive, if there exists a constant $k \in[0,1)$ such that

$$
\|B x-B y\|^{2} \leq\|x-y\|^{2}+k\|(I-B) x-(I-B) y\|^{2}, \quad \forall x, y \in C .
$$

Remark 1.1 (see [3, Remark 1.1 pages 135-136]). If $B: C \rightarrow H$ is a $L_{B}$-Lipschitz continuous and relaxed $(c, d)$-cocoercive mapping with $d>c L_{B}^{2}$ and $0<\tau<2\left(d-c L_{B}^{2}\right) / L_{B}^{2}$, then $I-\tau B$ satisfies the following:

$$
\|(I-\tau B) x-(I-\tau B) y\| \leq(1-\tau \xi)\|x-y\|, \quad \forall x, y \in C,
$$

where $\xi=\left(L_{B}^{2} / 2\right)\left[2\left(d-c L_{B}^{2}\right) / L_{B}^{2}-\tau\right]$.

Similarly, if $D: C \rightarrow H$ is $L_{D}$-Lipschitz continuous and relaxed $\left(c^{\prime}, d^{\prime}\right)$-cocoercive mapping with $d^{\prime}>c^{\prime} L_{D}^{2}$ and $0<\delta<2\left(d^{\prime}-c^{\prime} L_{D}^{2}\right) / L_{D}^{2}$, then the mapping $I-\delta D$ satisfies the following:

$$
\|(I-\delta D) x-(I-\delta D) y\| \leq\left(1-\delta \xi^{\prime}\right)\|x-y\|,
$$

where $\xi^{\prime}=\left(L_{D}^{2} / 2\right)\left[2\left(d^{\prime}-c^{\prime} L_{D}^{2}\right) / L_{D}^{2}-\delta\right]$.

Let $A$ be a strongly positive linear bounded operator on $H$ if there is a constant $\bar{\gamma}>0$ with the property

$$
\langle A x, x\rangle \geq \bar{\gamma}\|x\|^{2}, \quad \forall x \in H .
$$

We recall optimization problem (for short, OP) as the following

$$
\min _{x \in F} \frac{\mu}{2}\langle A x, x\rangle+\frac{1}{2}\|x-u\|^{2}-h(x)
$$

where $F=\cap_{n=1}^{\infty} C_{n}, C_{1}, C_{2}, \ldots$ are infinitely closed convex subsets of $H$ such that $\cap_{n=1}^{\infty} C_{n} \neq \emptyset$, $u \in H, \mu \geq 0$ is a real number, $A$ is a strongly positive linear bounded operator on $H$, and $h$ is a potential function for $\gamma f$ (i.e., $h^{\prime}(x)=\gamma f(x)$ for $x \in H$ ). This kind of optimization problem has been studied extensively by many authors, see, for example, [4-7] when $F=\cap_{n=1}^{\infty} C_{n}$ and $h(x)=\langle x, b\rangle$, where $b$ is a given point in $H$.

On the other hand, a family $\mathcal{S}=\{S(s): 0 \leq s<\infty\}$ of mappings of $C$ into itself is called a nonexpansive semigroup on $C$ if it satisfies the following conditions:

(i) $S(0) x=x$ for all $x \in C$;

(ii) $S(s+t)=S(s) S(t)$ for all $s, t \geq 0$;

(iii) $\|S(s) x-S(s) y\| \leq\|x-y\|$ for all $x, y \in C$ and $s \geq 0$;

(iv) for all $x \in C, s \mapsto S(s) x$ is continuous.

We denote by $F(\mathcal{S})$ the set of all common fixed points of $\mathcal{S}=\{S(s): s \geq 0\}$, that is, $F(\mathcal{S})=$ $\cap_{s \geq 0} F(S(s))$. It is known that $F(\mathcal{S})$ is closed and convex. 
Let $\phi: C \rightarrow \mathbb{R}$ be a real-valued function and let $\left\{\Theta_{k}: C \times C \rightarrow \mathbb{R}, k=1,2, \ldots, N\right\}$ be a finite family of equilibrium functions, that is, $\Theta_{k}(u, u)=0$ for each $u \in C$. The system of mixed equilibrium problems (for short, SMEP) for function $\left(\Theta_{1}, \Theta_{2}, \ldots, \Theta_{N}, \phi\right)$ is to find $z \in C$ such that

$$
\begin{array}{ll}
\Theta_{1}(z, y)+\phi(y)-\phi(z) \geq 0, & \forall y \in C, \\
\Theta_{2}(z, y)+\phi(y)-\phi(z) \geq 0, & \forall y \in C, \\
\vdots & \\
\Theta_{N}(z, y)+\phi(y)-\phi(z) \geq 0, & \forall y \in C .
\end{array}
$$

The set of solutions of (1.15) is denoted by $\cap_{k=1}^{N} \operatorname{MEP}\left(\Theta_{k}, \phi\right)$, where $\operatorname{MEP}\left(\Theta_{k}, \phi\right)$ is the set of solutions of the mixed equilibrium problem (for short, $\mathrm{MEP}$ ), which is to find $z \in C$ such that

$$
\Theta_{k}(z, y)+\phi(y)-\phi(z) \geq 0, \quad \forall y \in C
$$

In particular, if $\phi \equiv 0$, and $N=1$, then the problem (1.15) reduces to the equilibrium problem (for short, EP), which is to find $z \in C$ such that

$$
\Theta(z, y) \geq 0, \quad \forall y \in C
$$

It is well known that the SMEP includes fixed point problem, optimization problem, variational inequality problem, and Nash equilibrium problem as its special cases (see [813] for more details).

For solving the solutions of a nonexpansive semigroup and the solutions of the system of mixed equilibrium problems were studied by many authors see [14-23] and reference therein. In 2010, Chang et al. [24] studied the following approximation method:

$$
\begin{aligned}
& \Theta_{1}\left(u_{n}^{(1)}, x\right)+\phi(x)-\phi\left(u_{n}^{(1)}\right)+\frac{1}{r_{1}}\left\langle K^{\prime}\left(u_{n}^{(1)}\right)-K^{\prime}\left(x_{n}\right), \eta\left(x, u_{n}^{(1)}\right)\right\rangle \geq 0, \quad \forall x \in C, \\
& \Theta_{2}\left(u_{n}^{(2)}, x\right)+\phi(x)-\phi\left(u_{n}^{(2)}\right)+\frac{1}{r_{2}}\left\langle K^{\prime}\left(u_{n}^{(2)}\right)-K^{\prime}\left(x_{n}\right), \eta\left(x, u_{n}^{(2)}\right)\right\rangle \geq 0, \quad \forall x \in C, \\
& \vdots \\
& \Theta_{N}\left(u_{n}^{(N)}, x\right)+\phi(x)-\phi\left(u_{n}^{(N)}\right)+\frac{1}{r_{N}}\left\langle K^{\prime}\left(u_{n}^{(N)}\right)-K^{\prime}\left(x_{n}\right), \eta\left(x, u_{n}^{(N)}\right)\right\rangle \geq 0, \quad \forall x \in C, \\
& x_{n+1}=\alpha_{n} f\left(W_{n} x_{n}\right)+\beta_{n} x_{n}+\gamma_{n} \frac{1}{t_{n}} \int_{0}^{t_{n}} S(s) W_{n} u_{n}^{(N)} d s,
\end{aligned}
$$

where

$$
\begin{aligned}
u_{n}^{(1)} & =J_{r_{1}}^{\Theta_{1}} x_{n}, \\
u_{n}^{(k)} & =J_{r_{k}}^{\Theta_{k}} u_{n}^{(k-1)}=J_{r_{k}}^{\Theta_{k}} J_{r_{k-1}}^{\Theta_{k-1}} u_{n}^{(k-2)}=J_{r_{k}}^{\Theta_{k}} \cdots J_{r_{2}}^{\Theta_{2}} u_{n}^{(1)}, \\
& =J_{r_{k}}^{\Theta_{k}} \cdots J_{r_{2}}^{\Theta_{2}} J_{r_{1}}^{\Theta_{1}} x_{n}, \quad k=2,3, \ldots, N,
\end{aligned}
$$


$J_{r_{k}}^{\Theta_{k}}: C \rightarrow C, k=1,2, \ldots, N$ is the mapping defined by (2.22) below, $W_{n}$ is the mapping defined by (2.12), and $S=\{S(s): 0 \leq s<\infty\}$ is a nonexpansive semigroup. They proved that $\left\{x_{n}\right\}$ converges strongly to a fixed point of $F(\mathcal{S}) \cap F(W) \cap\left(\cap_{k=1}^{N} \operatorname{MEP}\left(\Theta_{k}, \phi\right)\right)$ under control conditions on the parameters.

Let $B, D: C \rightarrow H$ be two mappings. The general system of variational inequalities problem (see [25]) is to find $\left(x^{*}, y^{*}\right) \in C \times C$ such that

$$
\begin{array}{ll}
\left\langle\tau B y^{*}+x^{*}-y^{*}, x-x^{*}\right\rangle \geq 0, & \forall x \in C, \\
\left\langle\delta D x^{*}+y^{*}-x^{*}, x-y^{*}\right\rangle \geq 0, & \forall x \in C,
\end{array}
$$

where $\tau$ and $\delta$ are two positive real numbers. The set of solutions of the general system of variational inequalities problem is denoted by $\operatorname{SVI}(C, B, D)$. In particular, if $B=D$, then the problem (1.20) reduces to the following equation:

$$
\begin{array}{ll}
\left\langle\tau B y^{*}+x^{*}-y^{*}, x-x^{*}\right\rangle \geq 0, & \forall x \in C, \\
\left\langle\delta B x^{*}+y^{*}-x^{*}, x-y^{*}\right\rangle \geq 0, & \forall x \in C,
\end{array}
$$

which is defined by Verma [26] (see also Verma [27]), and is called the new system of variational inequalities. Further, if we set $D=0$, then problem (1.20) reduces to the classical variational inequality is to find $x^{*} \in C$ such that

$$
\left\langle B x^{*}, x-x^{*}\right\rangle \geq 0, \quad \forall x \in C \text {. }
$$

We denoted by $\operatorname{VI}(C, B)$ the set of solutions of the variational inequality problem. The variational inequality problem has been extensively studied in literature, see, for example, [28-31] and references therein. In order to find the solutions of the general system of variational inequality problem (1.20), Wangkeeree and Kamraksa [32] considered the following iterative algorithm:

$$
\begin{gathered}
\Theta\left(u_{n}, x\right)+\phi(x)-\phi\left(u_{n}\right)+\frac{1}{r}\left\langle K^{\prime}\left(u_{n}\right)-K^{\prime}\left(x_{n}\right), \eta\left(x, u_{n}\right)\right\rangle \geq 0, \quad \forall x \in C, \\
z_{n}=P_{C}\left(u_{n}-\delta D u_{n}\right), \\
\left.x_{n+1}=\alpha_{n} \gamma f\left(x_{n}\right)+\beta_{n} x_{n}+\left[\left(1-\beta_{n}\right) I-\alpha_{n} A\right)\right] W_{n} P_{C}\left(z_{n}-\tau B z_{n}\right),
\end{gathered}
$$

where $B, D: C \rightarrow H$ is a $L_{B}$-Lipschitz continuous and relaxed $(c, d)$-cocoercive mapping and $L_{D}$-Lipschitz continuous and relaxed $\left(c^{\prime}, d^{\prime}\right)$-cocoercive mapping, respectively. They proved that $\left\{x_{n}\right\}$ converges strongly to a fixed point of $F\left(W_{n}\right) \cap \operatorname{MEP}(\Theta, \phi) \cap \operatorname{SVI}(C, B, D)$ which is a solution of general system of variational inequality (1.20). Very recently, Jaiboon and Kumam [33] studied a new general iterative method for finding a common element of the set of solution of a mixed equilibrium problem, the set of fixed points of an infinite family of nonexpansive mappings, and the set of solutions of variational inequalities for an inversestrongly monotone mapping in Hilbert spaces, which solves some optimization problems.

Inspired and motivated by Chang et al. [24], Jaiboon and Kumam [33], Kumam and Jaiboon [34] and Wangkeeree and Kamraksa [32], the purpose of this paper is to introduce an iterative algorithm for finding a common element of the set of solutions of (1.15), the 
set of solutions of (1.20) for Lipschitz continuous and relaxed cocoercive mappings, the set of common fixed points for nonexpansive semigroup, and the set of common fixed points for an infinite family of strictly pseudocontractive mappings. Consequently, we prove the strong convergence theorem in Hilbert spaces under control conditions on the parameters. Furthermore, we can apply our results for solving some optimization problems. Our results extend and improve the corresponding results in Chang et al. [24], Kumam and Jaiboon [34], Wangkeeree and Kamraksa [32], and many others.

\section{Preliminaries}

Let $H$ a real Hilbert space and $C$ a nonempty closed convex subset of $H$. We denote strong convergence (weak convergence) by notation $\rightarrow(-)$. In a real Hilbert space $H$, it is well known that

$$
\begin{gathered}
\|x-y\|^{2}=\|x\|^{2}-\|y\|^{2}-2\langle x-y, y\rangle, \\
\|x+y\|^{2} \leq\|x\|^{2}+2\langle y, x+y\rangle, \\
\|x+y\|^{2} \geq\|x\|^{2}+2\langle y, x\rangle, \\
\|\lambda x+(1-\lambda) y\|^{2}=\lambda\|x\|^{2}+(1-\lambda)\|y\|^{2}-\lambda(1-\lambda)\|x-y\|^{2}
\end{gathered}
$$

for all $x, y \in H$ and $\lambda \in \mathbb{R}$.

Recall that for every point $x \in H$, there exists a unique nearest point in $C$, denoted by $P_{C} x$, such that

$$
\left\|x-P_{C} x\right\| \leq\|x-y\|, \quad \forall y \in C
$$

$P_{C}$ is called the metric projection of $H$ onto $C$. It is well known that $P_{C}$ is a nonexpansive mapping of $H$ onto $C$ and satisfies

$$
\left\langle x-y, P_{C} x-P_{C} y\right\rangle \geq\left\|P_{C} x-P_{C} y\right\|^{2}
$$

for every $x, y \in H$. Obviously, this immediately implies that

$$
\left\|(x-y)-\left(P_{C} x-P_{C} y\right)\right\|^{2} \leq\|x-y\|^{2}-\left\|P_{C} x-P_{C} y\right\|^{2}, \quad \forall x, y \in H .
$$

Moreover, $P_{C} x$ is characterized by the following properties: $P_{C} x \in C$ and

$$
\begin{gathered}
\left\langle x-P_{C} x, y-P_{C} x\right\rangle \leq 0, \\
\|x-y\|^{2} \geq\left\|x-P_{C} x\right\|^{2}+\left\|y-P_{C} x\right\|^{2}
\end{gathered}
$$

for all $x \in H, y \in C$. 
In order to prove our main results, we need the following lemmas.

Lemma 2.1 (see [35]). Let $V: C \rightarrow H$ be a $k$-strict pseudo-contraction, then

(1) the fixed point set $F(V)$ of $V$ is closed convex so that the projection $P_{F(V)}$ is well defined;

(2) define a mapping $T: C \rightarrow H$ by

$$
T x=t x+(1-t) V x, \quad \forall x \in C .
$$

If $t \in[k, 1)$, then $T$ is a nonexpansive mapping such that $F(V)=F(T)$.

A family of mappings $\left\{V_{i}: C \rightarrow H\right\}_{i=1}^{\infty}$ is called a family of uniformly $k$-strict pseudocontractions, if there exists a constant $k \in[0,1)$ such that

$$
\left\|V_{i} x-V_{i} y\right\|^{2} \leq\|x-y\|^{2}+k\left\|\left(I-V_{i}\right) x-\left(I-V_{i}\right) y\right\|^{2}, \quad \forall x, y \in C, \forall i \geq 1 .
$$

Let $\left\{V_{i}: C \rightarrow C\right\}_{i=1}^{\infty}$ be a countable family of uniformly $k$-strict pseudo-contractions. Let $\left\{T_{i}: C \rightarrow C\right\}_{i=1}^{\infty}$ be the sequence of nonexpansive mappings defined by (2.9), that is,

$$
T_{i} x=t x+(1-t) V_{i} x, \quad \forall x \in C, \forall i \geq 1, t \in[k, 1) .
$$

Let $\left\{T_{i}\right\}$ be a sequence of nonexpansive mappings of $C$ into itself defined by (2.11) and let $\left\{\mu_{i}\right\}$ be a sequence of nonnegative numbers in $[0,1]$. For each $n \geq 1$, define a mapping $W_{n}$ of $C$ into itself as follows:

$$
\begin{aligned}
U_{n, n+1} & =I, \\
U_{n, n} & =\mu_{n} T_{n} U_{n, n+1}+\left(1-\mu_{n}\right) I, \\
U_{n, n-1} & =\mu_{n-1} T_{n-1} U_{n, n}+\left(1-\mu_{n-1}\right) I, \\
& \vdots \\
U_{n, k} & =\mu_{k} T_{k} U_{n, k+1}+\left(1-\mu_{k}\right) I, \\
U_{n, k-1} & =\mu_{k-1} T_{k-1} U_{n, k}+\left(1-\mu_{k-1}\right) I, \\
& \vdots \\
U_{n, 2} & =\mu_{2} T_{2} U_{n, 3}+\left(1-\mu_{2}\right) I, \\
W_{n} & =U_{n, 1}=\mu_{1} T_{1} U_{n, 2}+\left(1-\mu_{1}\right) I .
\end{aligned}
$$

Such a mapping $W_{n}$ is nonexpansive from $C$ to $C$ and it is called the $W$-mapping generated by $T_{1}, T_{2}, \ldots, T_{n}$ and $\mu_{1}, \mu_{2}, \ldots, \mu_{n}$.

For each $n, k \in \mathbb{N}$, let the mapping $U_{n, k}$ be defined by (2.12). Then we can have the following crucial conclusions concerning $W_{n}$. You can find them in [36]. Now we only need the following similar version in Hilbert spaces.

Lemma 2.2 (see [36]). Let $C$ be a nonempty closed convex subset of a real Hilbert space H. Let $T_{1}, T_{2}, \ldots$ be nonexpansive mappings of $C$ into itself such that $\cap_{n=1}^{\infty} F\left(T_{n}\right)$ is nonempty, let $\mu_{1}, \mu_{2}, \ldots$ be real numbers such that $0 \leq \mu_{n} \leq b<1$ for every $n \geq 1$. Then, 
(1) $W_{n}$ is nonexpansive and $F\left(W_{n}\right)=\cap_{i=1}^{n} F\left(T_{i}\right)$, for all $n \geq 1$;

(2) for every $x \in C$ and $k \in \mathbb{N}$, the limit $\lim _{n \rightarrow \infty} U_{n, k} x$ exists;

(3) a mapping $W: C \rightarrow C$ defined by

$$
W x:=\lim _{n \rightarrow \infty} W_{n} x=\lim _{n \rightarrow \infty} U_{n, 1} x, \quad \forall x \in C
$$

is a nonexpansive mapping satisfying $F(W)=\cap_{i=1}^{\infty} F\left(T_{i}\right)$ and it is called the $W$-mapping generated by $T_{1}, T_{2}, \ldots$ and $\mu_{1}, \mu_{2}, \ldots$

Lemma 2.3 (see [37]). Let $C$ be a nonempty closed convex subset of a Hilbert space $H,\left\{T_{i}: C \rightarrow\right.$ $C\}$ a countable family of nonexpansive mappings with $\cap_{i=1}^{\infty} F\left(T_{i}\right) \neq \emptyset,\left\{\mu_{i}\right\}$ a real sequence such that $0<\mu_{i} \leq b<1$, for all $i \geq 1$. If $D$ is any bounded subset of $C$, then

$$
\lim _{n \rightarrow \infty} \sup _{x \in D}\left\|W x-W_{n} x\right\|=0 .
$$

Lemma 2.4 (see [38]). Each Hilbert space $H$ satisfies Opial's condition, that is, for any sequence $\left\{x_{n}\right\} \subset H$ with $x_{n} \rightarrow x$, the inequality

$$
\liminf _{n \rightarrow \infty}\left\|x_{n}-x\right\|<\liminf _{n \rightarrow \infty}\left\|x_{n}-y\right\|
$$

holds for each $y \in H$ with $y \neq x$.

Lemma 2.5 (see [39]). Assume $A$ is a strongly positive linear bounded operator on $H$ with coefficient $\bar{\gamma}>0$ and $0<\rho \leq\|A\|^{-1}$. Then, $\|I-\rho A\| \leq 1-\rho \bar{\gamma}$.

For solving the system of mixed equilibrium problems (1.15), let us assume that function $\Theta_{k}: H \times H \rightarrow \mathbb{R}, k=1,2, \ldots, N$ satisfies the following conditions:

(H1) $\Theta_{k}$ is monotone, that is, $\Theta_{k}(x, y)+\Theta_{k}(y, x) \leq 0$, for all $x, y \in H$;

(H2) for each fixed $y \in H, x \mapsto \Theta_{k}(x, y)$ is convex and upper semicontinuous;

(H3) for each $x \in H, y \mapsto \Theta_{k}(x, y)$ is convex.

Let $\eta: H \times H \rightarrow H$ and $B: H \rightarrow H$ be two mappings. $B$ is said to be

(1) monotone if

$$
\langle B x-B y, \eta(x, y)\rangle \geq 0, \quad \forall x, y \in H
$$

(2) $d$-strongly monotone if there exists a positive real number $d$ such that

$$
\langle B x-B y, \eta(x, y)\rangle \geq d\|x-y\|^{2}, \quad \forall x, y \in H
$$

(3) L-Lipschitz continuous if there exists a constant $L>0$ such that

$$
\|\eta(x, y)\| \leq L\|x-y\|, \quad \forall x, y \in H .
$$


Let $K: H \rightarrow \mathbb{R}$ be a differentiable functional on $H$, which is called:

(1) $\eta$-convex $[40]$ if

$$
K(y)-K(x) \geq\left\langle K^{\prime}(x), \eta(y, x)\right\rangle, \quad \forall x, y \in H
$$

where $K^{\prime}(x)$ is the Fréchet derivative of $K$ at $x$;

(2) $\eta$-strongly convex [41] if there exists a constant $\sigma>0$ such that

$$
K(y)-K(x)-\left\langle K^{\prime}(x), \eta(y, x)\right\rangle \geq \frac{\sigma}{2}\|x-y\|^{2}, \quad \forall x, y \in H .
$$

In particular, if $\eta(x, y)=x-y$ for all $x, y \in H$, then $K$ is said to be strongly convex.

Lemma 2.6 (see [42]). Let $H$ be a real Hilbert space and let $\phi$ be a lower semicontinuous and convex functional from $H$ to $\mathbb{R}$. Let $\Theta$ be a bifunction from $H \times H$ to $\mathbb{R}$ satisfying (H1)-(H3). Assume that

(i) $\eta: H \times H \rightarrow H$ is $\lambda$-Lipschitz continuous with constant $\lambda>0$ such that

(a) $\eta(x, y)+\eta(y, x)=0$, for all $x, y \in H$,

(b) $\eta(\cdot, \cdot)$ is affine in the first variable,

(c) for each fixed $x \in H, y \mapsto \eta(x, y)$ is sequentially continuous from the weak topology to the weak topology;

(ii) $K: H \rightarrow \mathbb{R}$ is $\eta$-strongly convex with constant $\sigma>0$ and its derivative $K^{\prime}$ is sequentially continuous from the weak topology to the strong topology;

(iii) for each $x \in H$, there exist bounded subsets $E_{x} \subset H$ and $z_{x} \in H$ such that for any $y \in H \backslash E_{x}$

$$
\Theta\left(y, z_{x}\right)+\phi\left(z_{x}\right)-\phi(y)+\frac{1}{r}\left\langle K^{\prime}(y)-K^{\prime}(x), \eta\left(z_{x}, y\right)\right\rangle<0 .
$$

For given $r>0$, let $J_{r}^{\Theta}: H \rightarrow H$ be the mapping defined by

$$
J_{r}^{\Theta}(x)=\left\{y \in H: \Theta(y, z)+\phi(z)-\phi(y)+\frac{1}{r}\left\langle K^{\prime}(y)-K^{\prime}(x), \eta(z, y)\right\rangle \geq 0, \forall z \in H\right\}
$$

for all $x \in H$. Then

(1) $J_{r}^{\Theta}$ is single-valued.

(2) $F\left(J_{r}^{\Theta}\right)=\operatorname{MEP}(\Theta, \Subset)$, where $\operatorname{MEP}(\Theta, \Subset)$ is the set of solution of the mixed equilibrium problem,

$$
\Theta(x, y)+\phi(y)-\phi(x) \geq 0, \quad \forall y \in H
$$

(3) $\operatorname{MEP}(\Theta,, E)$ is closed and convex. 
Lemma 2.7 (see [43]). Let $\left\{x_{n}\right\}$ and $\left\{v_{n}\right\}$ be bounded sequences in a Banach space $X$ and let $\left\{\beta_{n}\right\}$ be a sequence in $[0,1]$ with $0<\liminf _{n \rightarrow \infty} \beta_{n} \leq \lim \sup _{n \rightarrow \infty} \beta_{n}<1$. Suppose $x_{n+1}=\left(1-\beta_{n}\right) v_{n}+\beta_{n} x_{n}$ for all integers $n \geq 0$ and $\lim \sup _{n \rightarrow \infty}\left(\left\|v_{n+1}-v_{n}\right\|-\left\|x_{n+1}-x_{n}\right\|\right) \leq 0$. Then, $\lim _{n \rightarrow \infty}\left\|v_{n}-x_{n}\right\|=0$.

Lemma 2.8 (see [44]). Assume $\left\{x_{n}\right\}$ is a sequence of nonnegative real numbers such that

$$
x_{n+1} \leq\left(1-a_{n}\right) x_{n}+b_{n}, \quad \forall n \geq 0,
$$

where $\left\{a_{n}\right\}$ is a sequence in $(0,1)$ and $\left\{b_{n}\right\}$ is a sequence in $\mathbb{R}$ such that

(1) $\sum_{n=1}^{\infty} a_{n}=\infty$,

(2) $\lim \sup _{n \rightarrow \infty}\left(b_{n} / a_{n}\right) \leq 0$ or $\sum_{n=1}^{\infty}\left|b_{n}\right|<\infty$.

Then, $\lim _{n \rightarrow \infty} x_{n}=0$.

Lemma 2.9 (see [45]). Let $C$ be a nonempty closed convex subset of a real Hilbert space $H$ and $g: C \rightarrow \mathbb{R} \cup\{\infty\}$ a proper lower-semicontinuous differentiable convex function. If $z$ is a solution to the minimization problem

$$
g(z)=\inf _{x \in C} g(x)
$$

then

$$
\left\langle g^{\prime}(x), x-z\right\rangle \geq 0, \quad x \in C
$$

In particular, if $z$ solves problem $O P$, then

$$
\langle u+[\gamma f-(I+\mu A)] z, x-z\rangle \leq 0 .
$$

Lemma 2.10 (see [46]). Let $C$ be a nonempty bounded closed convex subset of a Hilbert space $H$ and let $\mathcal{S}=\{S(s): 0 \leq s<\infty\}$ be a nonexpansive semigroup on $C$, then for any $h \geq 0$,

$$
\lim _{t \rightarrow \infty} \sup _{x \in C}\left\|\frac{1}{t} \int_{0}^{t} T(s) x d s-T(h)\left(\frac{1}{t} \int_{0}^{t} T(s) x d s\right)\right\|=0 .
$$

Lemma 2.11 (see [47]). Let $C$ be a nonempty bounded closed convex subset of $H,\left\{x_{n}\right\}$ a sequence in $C$, and $\mathcal{S}=\{S(s): 0 \leq s<\infty\}$ a nonexpansive semigroup on $C$. If the following conditions are satisfied:

(i) $x_{n} \rightarrow z$;

(ii) $\limsup \sup _{s \rightarrow \infty} \lim \sup _{n \rightarrow \infty}\left\|S(s) x_{n}-x_{n}\right\|=0$, then $z \in \mathcal{S}$. 
Lemma 2.12 (see [25]). For given $x^{*}, y^{*} \in C$ and $\left(x^{*}, y^{*}\right)$ is a solution of the problem (1.20) if and only if $x^{*}$ is a fixed point of the mapping $G: C \rightarrow C$ is defined by

$$
G(x)=P_{C}\left[P_{C}(x-\delta D x)-\tau B P_{C}(x-\delta D x)\right], \quad \forall x \in H,
$$

where $y^{*}=P_{C}(x-\delta D x), \delta$ and $\tau$ are positive constants and $B, D: H \rightarrow H$ are two mappings.

Throughout this paper, the set of fixed points of the mapping $G$ is denoted by $\operatorname{SVI}(C, B, D)$.

Lemma 2.13 (see [32]). Let $G: C \rightarrow C$ be defined in Lemma 2.12. If $B: H \rightarrow H$ is a $L_{B}$-Lipschitzian and relaxed $(c, d)$-cocoercive mapping and $D: H \rightarrow H$ is a $L_{D}$-Lipschitz and relaxed $\left(c^{\prime}, d^{\prime}\right)$-cocoercive mapping where $\tau \leq 2\left(d-c L_{B}^{2}\right) / L_{B}^{2}$ and $\delta \leq 2\left(d^{\prime}-c^{\prime} L_{D}^{2}\right) / L_{D^{\prime}}^{2}$ then $G$ is nonexpansive.

Lemma 2.14 (demiclosedness principle [48]). Assume that $S$ is a nonexpansive self-mapping of a nonempty closed convex subset $C$ of a real Hilbert space $H$. If $S$ has a fixed point, then $I-S$ is demiclosed; that is, whenever $\left\{x_{n}\right\}$ is a sequence in $C$ converging weakly to some $x \in C$ (for short, $\left.x_{n} \rightarrow x \in C\right)$, and the sequence $\left\{(I-S) x_{n}\right\}$ converges strongly to some $y\left(\right.$ for short, $\left.(I-S) x_{n} \rightarrow y\right)$, it follows that $(I-S) x=y$. Here $I$ is the identity operator of $H$.

\section{Main Results}

In this section, we prove a strong convergence theorem of an iterative algorithm (3.1) for finding the solutions of a common element of the set of solutions of (1.15), the set of solutions of (1.20) for Lipschitz continuous and relaxed cocoercive mappings, the set of common fixed points for nonexpansive semigroups, and the set of common fixed points for an infinite family of strictly pseudocontractive mappings in a real Hilbert space.

Theorem 3.1. Let $C$ be a nonempty closed convex subset of a real Hilbert space $H$ which $C+C \subset C$ and let $f$ be a contraction of $C$ into itself with $\alpha \in(0,1)$. Let $\phi$ be a lower semicontinuous and convex functional from $H$ to $\mathbb{R}$ and let $\left\{\Theta_{k}: H \times H \rightarrow \mathbb{R}, k=1,2, \ldots, N\right\}$ be a finite family of equilibrium functions satisfying conditions (H1)-(H3). Let $\mathcal{S}=\{S(s): 0 \leq s<\infty\}$ be a nonexpansive semigroup on $C$ and let $\left\{t_{n}\right\}$ be a positive real divergent sequence. Let $\left\{V_{i}: C \rightarrow C\right\}_{i=1}^{\infty}$ be a countable family of uniformly $k$-strict pseudo-contractions, let $\left\{T_{i}: C \rightarrow C\right\}_{i=1}^{\infty}$ be the countable family of nonexpansive mappings defined by $T_{i} x=t x+(1-t) V_{i} x$, for all $x \in C$, for all $i \geq 1, t \in[k, 1)$, let $W_{n}$ be the $W$-mapping defined by (2.12), and let $W$ be a mapping defined by (2.13) with $F(W) \neq \emptyset$. Let $A$ be a strongly positive linear bounded operator on $H$ with coefficient $\bar{\gamma}>0$ and let $0<\gamma<(1+\mu \bar{\gamma}) / \alpha$, $B: H \rightarrow H$ be a $L_{B}$-Lipschitz continuous and relaxed $(c, d)$-cocoercive mapping with $d>c L_{B^{\prime}}^{2}$ and let $D: H \rightarrow H$ be a $L_{D}$-Lipschitz continuous and relaxed $\left(c^{\prime}, d^{\prime}\right)$-cocoercive mapping with $d^{\prime}>c^{\prime} L_{D}^{2}$. Suppose that $\Omega:=F(\mathcal{S}) \cap F(W) \cap \mathfrak{F} \cap \operatorname{SVI}(C, B, D) \neq \emptyset$, where $\mathfrak{F}=\left(\cap_{k=1}^{N} \operatorname{MEP}\left(\Theta_{k}, \phi\right)\right)$. Let $\mu>0, \gamma>0$ and $r_{k}>0, k=1,2, \ldots, N$, which are constants. For given $x_{1} \in H$ arbitrarily and fixed $u \in H$, suppose $\left\{x_{n}\right\},\left\{y_{n}\right\},\left\{z_{n}\right\}$ and $\left\{u_{n}^{(k)}\right\}, k=1,2, \ldots, N$ are the sequences generated iteratively by

$$
\Theta_{1}\left(u_{n}^{(1)}, x\right)+\phi(x)-\phi\left(u_{n}^{(1)}\right)+\frac{1}{r_{1}}\left\langle K^{\prime}\left(u_{n}^{(1)}\right)-K^{\prime}\left(x_{n}\right), \eta\left(x, u_{n}^{(1)}\right)\right\rangle \geq 0, \quad \forall x \in H
$$




$$
\begin{aligned}
& \Theta_{2}\left(u_{n}^{(2)}, x\right)+\phi(x)-\phi\left(u_{n}^{(2)}\right)+\frac{1}{r_{2}}\left\langle K^{\prime}\left(u_{n}^{(2)}\right)-K^{\prime}\left(x_{n}\right), \eta\left(x, u_{n}^{(2)}\right)\right\rangle \geq 0, \quad \forall x \in H, \\
& \vdots \\
& \Theta_{N}\left(u_{n}^{(N)}, x\right)+\phi(x)-\phi\left(u_{n}^{(N)}\right)+\frac{1}{r_{N}}\left\langle K^{\prime}\left(u_{n}^{(N)}\right)-K^{\prime}\left(x_{n}\right), \eta\left(x, u_{n}^{(N)}\right)\right\rangle \geq 0, \quad \forall x \in H, \\
& z_{n}=P_{C}\left(u_{n}^{(N)}-\delta D u_{n}^{(N)}\right), \\
& y_{n}=P_{C}\left(z_{n}-\tau B z_{n}\right), \\
& x_{n+1}=\alpha_{n}\left[u+\gamma f\left(W_{n} x_{n}\right)\right]+\beta_{n} x_{n}+\left[\left(1-\beta_{n}\right) I-\alpha_{n}(I+\mu A)\right] \frac{1}{t_{n}} \int_{0}^{t_{n}} S(s) W_{n} y_{n} d s,
\end{aligned}
$$

where

$$
\begin{aligned}
u_{n}^{(1)} & =J_{r_{1}}^{\Theta_{1}} x_{n} \\
u_{n}^{(k)} & =J_{r_{k}}^{\Theta_{k}} u_{n}^{(k-1)}=J_{r_{k}}^{\Theta_{k}} J_{r_{k-1}}^{\Theta_{k-1}} u_{n}^{(k-2)}=J_{r_{k}}^{\Theta_{k}} \cdots J_{r_{2}}^{\Theta_{2}} u_{n}^{(1)}, \\
& =J_{r_{k}}^{\Theta_{k}} \cdots J_{r_{2}}^{\Theta_{2}} J_{r_{1}}^{\Theta_{1}} x_{n}, \quad k=2,3, \ldots, N,
\end{aligned}
$$

$J_{r_{k}}^{\Theta_{k}}: H \rightarrow H, k=1,2, \ldots, N$ is the mapping defined by (2.22) and $\left\{\alpha_{n}\right\}$ and $\left\{\beta_{n}\right\}$ are two sequences in $(0,1)$ for all $n \in \mathbb{N}$. Assume the following conditions are satisfied:

(C1) $\eta: H \times H \rightarrow H$ is $\lambda$-Lipschitz continuous with constant $\lambda>0$ such that

(a) $\eta(x, y)+\eta(y, x)=0$, for all $x, y \in H$,

(b) $x \mapsto \eta(x, y)$ is affine,

(c) for each fixed $y \in H, y \mapsto \eta(x, y)$ is sequentially continuous from the weak topology to the weak topology;

(C2) $K: H \rightarrow \mathbb{R}$ is $\eta$-strongly convex with constant $\sigma>0$ and its derivative $K^{\prime}$ is not only sequentially continuous from the weak topology to the strong topology but also Lipschitz continuous with a Lipschitz constant $v>0$ such that $\sigma>\lambda \mathcal{\nu}$;

(C3) for each $k \in\{1,2, \ldots, N\}$ and for all $x \in H$, there exist bounded subsets $E_{x} \subset H$ and $z_{x} \in H$ such that for any $y \in H \backslash E_{x}$,

$$
\Theta_{k}\left(y, z_{x}\right)+\phi\left(z_{x}\right)-\phi(y)+\frac{1}{r_{k}}\left\langle K^{\prime}(y)-K^{\prime}(x), \eta\left(z_{x}, y\right)\right\rangle<0
$$

(C4) $\lim _{n \rightarrow \infty} \alpha_{n}=0$ and $\sum_{n=1}^{\infty} \alpha_{n}=\infty$;

(C5) $0<\liminf _{n \rightarrow \infty} \beta_{n} \leq \lim \sup _{n \rightarrow \infty} \beta_{n}<1$;

(C6) $0<\tau<2\left(d-c L_{B}^{2}\right) / L_{B}^{2}$ and $0<\delta<2\left(d^{\prime}-c^{\prime} L_{D}^{2}\right) / L_{D}^{2}$.

Then, $\left\{x_{n}\right\}$ converges strongly to $x^{*} \in \Omega$, which solves the following optimization problem (OP):

$$
\min _{x^{*} \in \Omega} \frac{\mu}{2}\left\langle A x^{*}, x^{*}\right\rangle+\frac{1}{2}\left\|x^{*}-u\right\|^{2}-h\left(x^{*}\right),
$$


and $\left(x^{*}, y^{*}\right)$ is a solution of the general system of variational inequality problem (1.20) such that $y^{*}=P_{C}\left(x^{*}-\delta D x^{*}\right)$.

Proof. By the condition (C4) and (C5), we may assume, without loss of generality, that $\alpha_{n} \leq$ $\left(1-\beta_{n}\right)(1+\mu\|A\|)^{-1}$ for all $n \in \mathbb{N}$. Indeed, $A$ is a strongly positive bounded linear operator on $H$, we have

$$
\|A\|=\sup \{|\langle A x, x\rangle|: x \in H,\|x\|=1\} .
$$

Observe that

$$
\begin{aligned}
\left\langle\left(\left(1-\beta_{n}\right) I-\alpha_{n}(I+\mu A)\right) x, x\right\rangle & =1-\beta_{n}-\alpha_{n}-\alpha_{n} \mu\langle A x, x\rangle \\
& \geq 1-\beta_{n}-\alpha_{n}-\alpha_{n} \mu\|A\| \\
& \geq 0
\end{aligned}
$$

so this shows that $\left(1-\beta_{n}\right) I-\alpha_{n}(I+\mu A)$ is positive. It follows that

$$
\begin{aligned}
\left\|\left(1-\beta_{n}\right) I-\alpha_{n}(I+\mu A)\right\| & =\sup \left\{\left|\left\langle\left(\left(1-\beta_{n}\right) I-\alpha_{n}(I+\mu A)\right) x, x\right\rangle\right|: x \in H,\|x\|=1\right\} \\
& =\sup \left\{1-\beta_{n}-\alpha_{n}-\alpha_{n} \mu\langle A x, x\rangle: x \in H,\|x\|=1\right\} \\
& \leq 1-\beta_{n}-\alpha_{n}-\alpha_{n} \mu \bar{\gamma} .
\end{aligned}
$$

We shall divide the proofs into several steps.

Step 1. We show that $\left\{x_{n}\right\}$ is bounded.

Let $x^{*} \in \Omega:=F(\mathcal{S}) \cap F(W) \cap\left(\cap_{k=1}^{N} \operatorname{MEP}\left(\Theta_{k}, \phi\right)\right) \cap \operatorname{SVI}(C, B, D)$. In fact, by the assumption that for each $k \in\{1,2, \ldots, N\}, J_{r_{k}}^{\Theta_{k}}$ is nonexpansive. Let $\mathscr{A}^{N}:=J_{r_{N}}^{\Theta_{N}} \cdots J_{r_{2}}^{\Theta_{2}} J_{r_{1}}^{\Theta_{1}}$ and $\mathscr{A}^{0}=I$. Then, we have $x^{*}=\mathscr{A}^{N} x^{*}$ and $u_{n}^{(N)}=\mathscr{A}^{N} x_{n}$. Since $x^{*} \in \operatorname{SVI}(C, B, D)$, then

$$
x^{*}=P_{C}\left[P_{C}\left(x^{*}-\delta D x^{*}\right)-\tau B P_{C}\left(x^{*}-\delta D x^{*}\right)\right]=P_{C}\left[P_{C}(I-\delta D) \mathcal{A}^{N} x^{*}-\tau B P_{C}(I-\delta D) \mathscr{A}^{N} x^{*}\right] .
$$

Putting $y^{*}=P_{C}\left(x^{*}-\delta D x^{*}\right)=P_{C}(I-\delta D) \mathcal{A}^{N} x^{*}$, we have $x^{*}=P_{C}\left(y^{*}-\tau B y^{*}\right)$. Since $x^{*}=$ $S(s) x^{*}$, for all $s \geq 0$ and $x^{*}=W_{n} x^{*}$, for all $n \geq 1$, therefore, we have

$$
x^{*}=\mathcal{A}^{N} x^{*}=P_{C}\left(y^{*}-\tau B y^{*}\right)=W_{n} P_{C}\left(y^{*}-\tau B y^{*}\right)=S(s) W_{n} P_{C}\left(y^{*}-\tau B y^{*}\right) .
$$

Because $P_{C}$ and $\mathscr{A}^{N}$ are nonexpansive mappings and from Remark 1.1, we have

$$
\begin{aligned}
\left\|y_{n}-x^{*}\right\| & =\left\|P_{C}\left(z_{n}-\tau B z_{n}\right)-P_{C}\left(y^{*}-\tau B y^{*}\right)\right\| \\
& \leq\left\|(I-\tau B) z_{n}-(I-\tau B) y^{*}\right\| \\
& \leq\left\|z_{n}-y^{*}\right\| \\
& =\left\|P_{C}\left(u_{n}^{(N)}-\delta D u_{n}^{(N)}\right)-P_{C}\left(x^{*}-\delta D x^{*}\right)\right\|
\end{aligned}
$$




$$
\begin{aligned}
& \leq\left\|(I-\delta D) u_{n}^{(N)}-(I-\delta D) x^{*}\right\| \\
& \leq\left\|u_{n}^{(N)}-x^{*}\right\| \\
& =\left\|\mathscr{A}^{N} x_{n}-\mathcal{A}^{N} x^{*}\right\| \\
& \leq\left\|x_{n}-x^{*}\right\|
\end{aligned}
$$

which yields that

$$
\begin{aligned}
\left\|x_{n+1}-x^{*}\right\|= & \| \alpha_{n} u+\alpha_{n}\left(\gamma f\left(W_{n} x_{n}\right)-(I+\mu A) x^{*}\right)+\beta_{n}\left(x_{n}-x^{*}\right) \\
& +\left(\left(1-\beta_{n}\right) I-\alpha_{n}(I+\mu A)\right)\left(\frac{1}{t_{n}} \int_{0}^{t_{n}} S(s) W_{n} y_{n} d s-x^{*}\right) \| \\
\leq & \alpha_{n}\|u\|+\alpha_{n}\left\|\gamma f\left(W_{n} x_{n}\right)-(I+\mu A) x^{*}\right\|+\beta_{n}\left\|x_{n}-x^{*}\right\| \\
& +\left(1-\beta_{n}-\alpha_{n}(1+\mu \bar{\gamma})\right)\left\|x_{n}-x^{*}\right\| \\
\leq & \alpha_{n}\|u\|+\alpha_{n}\left\|\gamma f\left(W_{n} x_{n}\right)-\gamma f\left(x^{*}\right)\right\|+\alpha_{n}\left\|\gamma f\left(x^{*}\right)-(I+\mu A) x^{*}\right\|+\beta_{n}\left\|x_{n}-x^{*}\right\| \\
& +\left(1-\beta_{n}-\alpha_{n}(1+\mu \bar{\gamma})\right)\left\|x_{n}-x^{*}\right\| \\
\leq & \alpha_{n}\|u\|+\alpha_{n} \gamma \alpha\left\|x_{n}-x^{*}\right\|+\alpha_{n}\left\|\gamma f\left(x^{*}\right)-(I+\mu A) x^{*}\right\|+\beta_{n}\left\|x_{n}-x^{*}\right\| \\
& +\left(1-\beta_{n}-\alpha_{n}(1+\mu \bar{\gamma})\right)\left\|x_{n}-x^{*}\right\| \\
= & \alpha_{n}\left(\|u\|+\left\|\gamma f\left(x^{*}\right)-(I+\mu A) x^{*}\right\|\right)+\left(1-\alpha_{n}(1+\mu \bar{\gamma})+\alpha_{n} \gamma \alpha\right)\left\|x_{n}-x^{*}\right\| \\
= & \left(1-\alpha_{n}((1+\mu \bar{\gamma})-\gamma \alpha)\right)\left\|x_{n}-x^{*}\right\| \\
& +\alpha_{n}((1+\mu \bar{\gamma})-\gamma \alpha) \frac{\|u\|+\left\|\gamma f\left(x^{*}\right)-(I+\mu A) x^{*}\right\|}{(1+\mu \bar{\gamma})-\gamma \alpha} .
\end{aligned}
$$

It follows from (3.11) and induction that

$$
\left\|x_{n}-x^{*}\right\| \leq \max \left\{\left\|x_{1}-p\right\|, \frac{\|u\|+\left\|\gamma f\left(x^{*}\right)-(I+\mu A) x^{*}\right\|}{(1+\mu \bar{\gamma})-\gamma \alpha}\right\}, \quad n \geq 1 .
$$

Hence, $\left\{x_{n}\right\}$ is bounded, so are $\left\{y_{n}\right\},\left\{z_{n}\right\},\left\{W_{n} x_{n}\right\},\left\{f\left(W_{n} x_{n}\right)\right\},\left\{u_{n}^{(k)}\right\}$ for all $k=1,2, \ldots, N$ and $\left\{K_{n} W_{n} y_{n}\right\}$, where $K_{n}=\left(1 / t_{n}\right) \int_{0}^{t_{n}} S(s) d s$.

Step 2. We prove that $\lim _{n \rightarrow \infty}\left\|x_{n+1}-x_{n}\right\|=0$ and $\lim _{n \rightarrow \infty}\left\|u_{n+1}^{(N)}-u_{n}^{(N)}\right\|=0$.

Again, from Remark 1.1, we have the following estimates:

$$
\begin{aligned}
\left\|y_{n+1}-y_{n}\right\| & =\left\|P_{C}\left(z_{n+1}-\tau B z_{n+1}\right)-P_{C}\left(z_{n}-\tau B z_{n}\right)\right\| \\
& \leq\left\|\left(z_{n+1}-\tau B z_{n+1}\right)-\left(z_{n}-\tau B z_{n}\right)\right\| \\
& \leq\left\|z_{n+1}-z_{n}\right\| \\
& =\left\|P_{C}\left(u_{n+1}^{(N)}-\delta D u_{n+1}^{(N)}\right)-P_{C}\left(u_{n}^{(N)}-\delta D u_{n}^{(N)}\right)\right\|
\end{aligned}
$$


Journal of Applied Mathematics

$$
\begin{aligned}
& \leq\left\|\left(u_{n+1}^{(N)}-\delta D u_{n+1}^{(N)}\right)-\left(u_{n}^{(N)}-\delta D u_{n}^{(N)}\right)\right\| \\
& \leq\left\|u_{n+1}^{(N)}-u_{n}^{(N)}\right\| \\
& =\left\|\mathscr{A}^{N} x_{n+1}-\mathcal{A}^{N} x_{n}\right\| \\
& \leq\left\|x_{n+1}-x_{n}\right\| .
\end{aligned}
$$

On the other hand, since $T_{i}$ and $U_{n, i}$ are nonexpansive, we have

$$
\begin{aligned}
\left\|W_{n+1} y_{n}-W_{n} y_{n}\right\| & =\left\|\mu_{1} T_{1} U_{n+1,2} y_{n}-\mu_{1} T_{1} U_{n, 2} y_{n}\right\| \\
& \leq \mu_{1}\left\|U_{n+1,2} y_{n}-U_{n, 2} y_{n}\right\| \\
& =\mu_{1}\left\|\mu_{2} T_{2} U_{n+1,3} y_{n}-\mu_{2} T_{2} U_{n, 3} y_{n}\right\| \\
& \leq \mu_{1} \mu_{2}\left\|U_{n+1,3} y_{n}-U_{n, 3} y_{n}\right\| \\
& \vdots \\
& \leq \mu_{1} \mu_{2} \cdots \mu_{n}\left\|U_{n+1, n+1} y_{n}-U_{n, n+1} y_{n}\right\| \\
& \leq M_{1} \prod_{i=1}^{n} \mu_{i},
\end{aligned}
$$

where $M_{1} \geq 0$ is a constant such that $\left\|U_{n+1, n+1} y_{n}-U_{n, n+1} y_{n}\right\| \leq M_{1}$ for all $n \geq 0$. It follows from (3.13) and (3.14) that we have

$$
\begin{aligned}
\left\|W_{n+1} y_{n+1}-W_{n} y_{n}\right\| & \leq\left\|W_{n+1} y_{n+1}-W_{n+1} y_{n}\right\|+\left\|W_{n+1} y_{n}-W_{n} y_{n}\right\| \\
& \leq\left\|y_{n+1}-y_{n}\right\|+M_{1} \prod_{i=1}^{n} \mu_{i} \\
& \leq\left\|x_{n+1}-x_{n}\right\|+M_{1} \prod_{i=1}^{n} \mu_{i}
\end{aligned}
$$

It follows that

$$
\begin{aligned}
& \| K_{n+1} W_{n+1} y_{n+1}-K_{n} W_{n} y_{n}\|=\| \frac{1}{t_{n+1}} \int_{0}^{t_{n+1}} S(s) W_{n+1} y_{n+1} d s-\frac{1}{t_{n}} \int_{0}^{t_{n}} S(s) W_{n} y_{n} d s \| \\
& \leq \frac{1}{t_{n+1}} \int_{0}^{t_{n+1}}\left\|S(s) W_{n+1} y_{n+1}-S(s) W_{n} y_{n}\right\| d s \\
& \quad+\left\|\frac{1}{t_{n+1}} \int_{0}^{t_{n+1}} S(s) W_{n} y_{n} d s-\frac{1}{t_{n}} \int_{0}^{t_{n}} S(s) W_{n} y_{n} d s\right\| \\
& \leq\left\|W_{n+1} y_{n+1}-W_{n} y_{n}\right\|+\left\|\frac{1}{t_{n+1}} \int_{t_{n}}^{t_{n+1}} S(s) W_{n} y_{n} d s+\frac{1}{t_{n+1}} \int_{0}^{t_{n}} S(s) W_{n} y_{n} d s-\frac{1}{t_{n}} \int_{0}^{t_{n}} S(s) W_{n} y_{n} d s\right\| \\
& \leq\left\|W_{n+1} y_{n+1}-W_{n} y_{n}\right\|+\frac{1}{t_{n+1}} \int_{t_{n}}^{t_{n+1}}\left\|S(s) W_{n} y_{n}\right\| d s
\end{aligned}
$$




$$
\begin{aligned}
& +\left|\frac{1}{t_{n+1}}-\frac{1}{t_{n}}\right| \int_{0}^{t_{n}}\left\|S(s) W_{n} y_{n}\right\| d s \\
\leq & \left\|W_{n+1} y_{n+1}-W_{n} y_{n}\right\|+2\left(1-\frac{t_{n}}{t_{n+1}}\right) M_{2} \\
\leq & \left\|x_{n+1}-x_{n}\right\|+M_{1} \prod_{i=1}^{n} \mu_{i}+2\left(1-\frac{t_{n}}{t_{n+1}}\right) M_{2}
\end{aligned}
$$

where $M_{2}=\max \left\{\left\|S(s) W_{n} y_{n}\right\|\right\}$.

Setting $x_{n+1}=\left(1-\beta_{n}\right) v_{n}+\beta_{n} x_{n}$, for all $n \geq 1$, we have

$$
v_{n}=\frac{x_{n+1}-\beta_{n} x_{n}}{1-\beta_{n}}=\frac{\alpha_{n}\left(u+\gamma f\left(W_{n} x_{n}\right)\right)+\left(\left(1-\beta_{n}\right) I-\alpha_{n}(I+\mu A)\right) K_{n} W_{n} y_{n}}{1-\beta_{n}}
$$

Then, we obtain

$$
\begin{aligned}
v_{n+1}-v_{n}= & \frac{\alpha_{n+1}\left(u+\gamma f\left(W_{n+1} x_{n+1}\right)\right)+\left(\left(1-\beta_{n+1}\right) I-\alpha_{n+1}(I+\mu A)\right) K_{n+1} W_{n+1} y_{n+1}}{1-\beta_{n+1}} \\
& -\frac{\alpha_{n}\left(u+\gamma f\left(W_{n} x_{n}\right)\right)+\left(\left(1-\beta_{n}\right) I-\alpha_{n}(I+\mu A)\right) K_{n} W_{n} y_{n}}{1-\beta_{n}} \\
= & \frac{\alpha_{n+1}}{1-\beta_{n+1}}\left(u+\gamma f\left(W_{n+1} x_{n+1}\right)\right)-\frac{\alpha_{n}}{1-\beta_{n}}\left(u+\gamma f\left(W_{n} x_{n}\right)\right)+K_{n+1} W_{n+1} y_{n+1}-K_{n} W_{n} y_{n} \\
& +\frac{\alpha_{n}}{1-\beta_{n}}(I+\mu A) K_{n} W_{n} y_{n}-\frac{\alpha_{n+1}}{1-\beta_{n+1}}(I+\mu A) K_{n+1} W_{n+1} y_{n+1} \\
= & \frac{\alpha_{n+1}}{1-\beta_{n+1}}\left(\left(u+\gamma f\left(W_{n+1} x_{n+1}\right)\right)-(I+\mu A) K_{n+1} W_{n+1} y_{n+1}\right) \\
& +\frac{\alpha_{n}}{1-\beta_{n}}\left((I+\mu A) K_{n} W_{n} y_{n}-u-\gamma f\left(W_{n} x_{n}\right)\right)+K_{n+1} W_{n+1} y_{n+1}-K_{n} W_{n} y_{n} .
\end{aligned}
$$

It follows from (3.16) and (3.18) that

$$
\begin{aligned}
\left\|v_{n+1}-v_{n}\right\|-\left\|x_{n+1}-x_{n}\right\| \leq & \frac{\alpha_{n+1}}{1-\beta_{n+1}}\left(\|u\|+\left\|\gamma f\left(W_{n+1} x_{n+1}\right)\right\|+\left\|(I+\mu A) K_{n+1} W_{n+1} y_{n+1}\right\|\right) \\
& +\frac{\alpha_{n}}{1-\beta_{n}}\left(\left\|(I+\mu A) K_{n} W_{n} y_{n}\right\|+\|u\|+\left\|\gamma f\left(W_{n} x_{n}\right)\right\|\right) \\
& +M_{1} \prod_{i=1}^{n} \mu_{i}+2\left(1-\frac{t_{n}}{t_{n+1}}\right) M_{2}
\end{aligned}
$$

By the conditions (C4), (C5) and from $t_{n} \in(0, \infty), t_{n} \rightarrow \infty$ and $0<\mu_{i} \leq b<1$, for all $i \geq 1$, we have

$$
\limsup _{n \rightarrow \infty}\left(\left\|v_{n+1}-v_{n}\right\|-\left\|x_{n+1}-x_{n}\right\|\right) \leq 0
$$


Journal of Applied Mathematics

Hence, by Lemma 2.7, we obtain

$$
\lim _{n \rightarrow \infty}\left\|v_{n}-x_{n}\right\|=0
$$

It follows that

$$
\lim _{n \rightarrow \infty}\left\|x_{n+1}-x_{n}\right\|=\lim _{n \rightarrow \infty}\left(1-\beta_{n}\right)\left\|v_{n}-x_{n}\right\|=0
$$

Applying (3.22) into (3.13), we obtain that

$$
\lim _{n \rightarrow \infty}\left\|y_{n+1}-y_{n}\right\|=\lim _{n \rightarrow \infty}\left\|z_{n+1}-z_{n}\right\|=\lim _{n \rightarrow \infty}\left\|u_{n+1}^{(N)}-u_{n}^{(N)}\right\|=0
$$

Step 3. We show that $\lim _{n \rightarrow \infty}\left\|K_{n} W_{n} y_{n}-y_{n}\right\|=0, \lim _{n \rightarrow \infty}\left\|y_{n}-S(s) y_{n}\right\|=0$, and $\lim _{n \rightarrow \infty}\left\|u_{n}^{(k+1)}-u_{n}^{(k)}\right\|=0$, where $K_{n}=\left(1 / t_{n}\right) \int_{0}^{t_{n}} S(s) d s$.

Since $x_{n+1}=\alpha_{n}\left(u+\gamma f\left(W_{n} x_{n}\right)\right)+\beta_{n} x_{n}+\left(\left(1-\beta_{n}\right) I-\alpha_{n}(I+\mu A)\right) K_{n} W_{n} y_{n}$, we have

$$
\begin{aligned}
\| x_{n}- & K_{n} W_{n} y_{n} \| \\
\leq & \left\|x_{n}-x_{n+1}\right\|+\left\|x_{n+1}-K_{n} W_{n} y_{n}\right\| \\
= & \left\|x_{n}-x_{n+1}\right\| \\
& +\left\|\alpha_{n}\left(u+\gamma f\left(W_{n} x_{n}\right)\right)+\beta_{n} x_{n}+\left(\left(1-\beta_{n}\right) I-\alpha_{n}(I+\mu A)\right) K_{n} W_{n} y_{n}-K_{n} W_{n} y_{n}\right\| \\
= & \left\|x_{n}-x_{n+1}\right\|+\left\|\alpha_{n}\left(\left(u+\gamma f\left(W_{n} x_{n}\right)\right)-(I+\mu A) K_{n} W_{n} y_{n}\right)+\beta_{n}\left(x_{n}-K_{n} W_{n} y_{n}\right)\right\| \\
\leq & \left\|x_{n}-x_{n+1}\right\|+\alpha_{n}\left(\|u\|+\left\|\gamma f\left(W_{n} x_{n}\right)\right\|+\left\|(I+\mu A) K_{n} W_{n} y_{n}\right\|\right) \\
& +\beta_{n}\left\|x_{n}-K_{n} W_{n} y_{n}\right\|,
\end{aligned}
$$

that is

$$
\left\|x_{n}-K_{n} W_{n} y_{n}\right\| \leq \frac{1}{1-\beta_{n}}\left\|x_{n}-x_{n+1}\right\|+\frac{\alpha_{n}}{1-\beta_{n}}\left(\|u\|+\left\|\gamma f\left(W_{n} x_{n}\right)\right\|+\left\|(I+\mu A) K_{n} W_{n} y_{n}\right\|\right)
$$

By (C4), (C5), and (3.22) it follows that

$$
\lim _{n \rightarrow \infty}\left\|K_{n} W_{n} y_{n}-x_{n}\right\|=0
$$


Since $J_{r_{N}}^{\Theta_{N}}: C \rightarrow C$ is firmly nonexpansive, $u_{n}^{(N)}=\mathcal{A}^{N} x_{n}$, where $\mathcal{A}^{N}:=J_{r_{N}}^{\Theta_{N}} \cdots J_{r_{2}}^{\Theta_{2}} J_{r_{1}}^{\Theta_{1}}$ and $x^{*} \in \Omega$, we have

$$
\begin{aligned}
\left\|u_{n}^{(N)}-x^{*}\right\|^{2} & =\left\|\mathcal{A}^{N} x_{n}-\mathcal{A}^{N} x^{*}\right\|^{2} \\
& \leq\left\langle\mathcal{A}^{N} x_{n}-\mathcal{A}^{N} x^{*}, x_{n}-x^{*}\right\rangle \\
& =\left\langle u_{n}^{(N)}-x^{*}, x_{n}-x^{*}\right\rangle \\
& =\frac{1}{2}\left(\left\|u_{n}^{(N)}-x^{*}\right\|^{2}+\left\|x_{n}-x^{*}\right\|^{2}-\left\|x_{n}-u_{n}^{(N)}\right\|^{2}\right),
\end{aligned}
$$

and hence

$$
\left\|u_{n}^{(N)}-x^{*}\right\|^{2} \leq\left\|x_{n}-x^{*}\right\|^{2}-\left\|x_{n}-u_{n}^{(N)}\right\|^{2}
$$

Observe that

$$
\begin{aligned}
\left\|x_{n+1}-x^{*}\right\|^{2}= & \|\left(\left(1-\beta_{n}\right) I-\alpha_{n}(I+\mu A)\right)\left(K_{n} W_{n} y_{n}-x^{*}\right)+\beta_{n}\left(x_{n}-x^{*}\right) \\
& +\alpha_{n}\left(u+\gamma f\left(W_{n} x_{n}\right)-(I+\mu A) x^{*}\right) \|^{2} \\
= & \left\|\left(\left(1-\beta_{n}\right) I-\alpha_{n}(I+\mu A)\right)\left(K_{n} W_{n} y_{n}-x^{*}\right)+\beta_{n}\left(x_{n}-x^{*}\right)\right\|^{2} \\
& +\alpha_{n}^{2}\left\|u+\gamma f\left(W_{n} x_{n}\right)-(I+\mu A) x^{*}\right\|^{2} \\
& +2 \beta_{n} \alpha_{n}\left\langle x_{n}-x^{*}, u+\gamma f\left(W_{n} x_{n}\right)-(I+\mu A) x^{*}\right\rangle \\
& +2 \alpha_{n}\left\langle\left(\left(1-\beta_{n}\right) I-\alpha_{n}(I+\mu A)\right)\left(K_{n} W_{n} y_{n}-x^{*}\right), u+\gamma f\left(W_{n} x_{n}\right)-(I+\mu A) x^{*}\right\rangle \\
\leq & {\left[\left(1-\beta_{n}-\alpha_{n}-\alpha_{n} \mu \bar{\gamma}\right)\left\|K_{n} W_{n} y_{n}-x^{*}\right\|+\beta_{n}\left\|x_{n}-x^{*}\right\|\right]^{2} } \\
& +\alpha_{n}^{2}\left\|u+\gamma f\left(W_{n} x_{n}\right)-(I+\mu A) x^{*}\right\|^{2} \\
& +2 \beta_{n} \alpha_{n}\left\langle x_{n}-x^{*}, u+\gamma f\left(W_{n} x_{n}\right)-(I+\mu A) x^{*}\right\rangle \\
& +2 \alpha_{n}\left\langle\left(\left(1-\beta_{n}\right) I-\alpha_{n}(I+\mu A)\right)\left(K_{n} W_{n} y_{n}-x^{*}\right), u+\gamma f\left(W_{n} x_{n}\right)-(I+\mu A) x^{*}\right\rangle \\
= & {\left[\left(1-\beta_{n}-\alpha_{n}-\alpha_{n} \mu \bar{\gamma}\right)\left\|K_{n} W_{n} y_{n}-x^{*}\right\|+\beta_{n}\left\|x_{n}-x^{*}\right\|\right]^{2}+c_{n} } \\
\leq & \left(1-\beta_{n}-\alpha_{n}-\alpha_{n} \mu \bar{\gamma}\right)^{2}\left\|K_{n} W_{n} y_{n}-x^{*}\right\|^{2}+\beta_{n}^{2}\left\|x_{n}-x^{*}\right\|^{2} \\
& +2\left(1-\beta_{n}-\alpha_{n}-\alpha_{n} \mu \bar{\gamma}\right) \beta_{n}\left\|K_{n} W_{n} y_{n}-x^{*}\right\|\left\|x_{n}-x^{*}\right\|+c_{n} \\
\leq & \left(1-\beta_{n}-\alpha_{n}-\alpha_{n} \mu \bar{\gamma}\right)^{2}\left\|K_{n} W_{n} y_{n}-x^{*}\right\|^{2}+\beta_{n}^{2}\left\|x_{n}-x^{*}\right\|^{2} \\
& +\left(1-\beta_{n}-\alpha_{n}-\alpha_{n} \mu \bar{\gamma}\right) \beta_{n}\left[\left\|K_{n} W_{n} y_{n}-x^{*}\right\|^{2}+\left\|x_{n}-x^{*}\right\|^{2}\right]+c_{n} \\
= & \left.\left(1-\alpha_{n}-\alpha_{n} \mu \bar{\gamma}\right)^{2}-2\left(1-\alpha_{n}-\alpha_{n} \mu \bar{\gamma}\right) \beta_{n}+\beta_{n}^{2}\right]\left\|K_{n} W_{n} y_{n}-x^{*}\right\|^{2}+\beta_{n}^{2}\left\|x_{n}-x^{*}\right\|^{2} \\
& +\left[\left(1-\alpha_{n}-\alpha_{n} \mu \bar{\gamma}\right) \beta_{n}-\beta_{n}^{2}\right]\left[\left\|K_{n} W_{n} y_{n}-x^{*}\right\|^{2}+\left\|x_{n}-x^{*}\right\|^{2}\right]+c_{n} \\
& {[(1+\mu)}
\end{aligned}
$$


Journal of Applied Mathematics

$$
\begin{aligned}
= & {\left[\left(1-\alpha_{n}-\alpha_{n} \mu \bar{\gamma}\right)^{2}-\left(1-\alpha_{n}-\alpha_{n} \mu \bar{\gamma}\right) \beta_{n}\right]\left\|K_{n} W_{n} y_{n}-x^{*}\right\|^{2} } \\
& +\left(1-\alpha_{n}-\alpha_{n} \mu \bar{\gamma}\right) \beta_{n}\left\|x_{n}-x^{*}\right\|^{2}+c_{n} \\
\leq & \left(1-\alpha_{n}-\alpha_{n} \mu \bar{\gamma}\right)\left(1-\beta_{n}-\alpha_{n}-\alpha_{n} \mu \bar{\gamma}\right)\left\|y_{n}-x^{*}\right\|^{2} \\
& +\left(1-\alpha_{n}-\alpha_{n} \mu \bar{\gamma}\right) \beta_{n}\left\|x_{n}-x^{*}\right\|^{2}+c_{n},
\end{aligned}
$$

where

$$
\begin{aligned}
c_{n}= & \alpha_{n}^{2}\left\|u+\gamma f\left(W_{n} x_{n}\right)-(I+\mu A) x^{*}\right\|^{2}+2 \beta_{n} \alpha_{n}\left\langle x_{n}-x^{*}, u+\gamma f\left(W_{n} x_{n}\right)-(I+\mu A) x^{*}\right\rangle \\
& +2 \alpha_{n}\left\langle\left(\left(1-\beta_{n}\right) I-\alpha_{n}(I+\mu A)\right)\left(K_{n} W_{n} y_{n}-x^{*}\right), u+\gamma f\left(W_{n} x_{n}\right)-(I+\mu A) x^{*}\right\rangle .
\end{aligned}
$$

It follows from condition (C4) that

$$
\lim _{n \rightarrow \infty} c_{n}=0 .
$$

Putting (3.28) into (3.29) and using also (3.10), we have

$$
\begin{aligned}
\left\|x_{n+1}-x^{*}\right\|^{2} \leq & \left(1-\alpha_{n}-\alpha_{n} \mu \bar{\gamma}\right)\left(1-\beta_{n}-\alpha_{n}-\alpha_{n} \mu \bar{\gamma}\right)\left\|y_{n}-x^{*}\right\|^{2} \\
& +\left(1-\alpha_{n}-\alpha_{n} \mu \bar{\gamma}\right) \beta_{n}\left\|x_{n}-x^{*}\right\|^{2}+c_{n} \\
\leq & \left(1-\alpha_{n}-\alpha_{n} \mu \bar{\gamma}\right)\left(1-\beta_{n}-\alpha_{n}-\alpha_{n} \mu \bar{\gamma}\right)\left\|u_{n}^{(N)}-x^{*}\right\|^{2} \\
& +\left(1-\alpha_{n}-\alpha_{n} \mu \bar{\gamma}\right) \beta_{n}\left\|x_{n}-x^{*}\right\|^{2}+c_{n} \\
\leq & \left(1-\alpha_{n}-\alpha_{n} \mu \bar{\gamma}\right)\left(1-\beta_{n}-\alpha_{n}-\alpha_{n} \mu \bar{\gamma}\right)\left\{\left\|x_{n}-x^{*}\right\|^{2}-\left\|x_{n}-u_{n}^{(N)}\right\|^{2}\right\} \\
& +\left(1-\alpha_{n}-\alpha_{n} \mu \bar{\gamma}\right) \beta_{n}\left\|x_{n}-x^{*}\right\|^{2}+c_{n} \\
= & \left(1-\alpha_{n}-\alpha_{n} \mu \bar{\gamma}\right)^{2}\left\|x_{n}-x^{*}\right\|^{2} \\
& -\left(1-\alpha_{n}-\alpha_{n} \mu \bar{\gamma}\right)\left(1-\beta_{n}-\alpha_{n}-\alpha_{n} \mu \bar{\gamma}\right)\left\|x_{n}-u_{n}^{(N)}\right\|^{2}+c_{n} \\
\leq & \left\|x_{n}-x^{*}\right\|^{2}-\left(1-\alpha_{n}-\alpha_{n} \mu \bar{\gamma}\right)\left(1-\beta_{n}-\alpha_{n}-\alpha_{n} \mu \bar{\gamma}\right)\left\|x_{n}-u_{n}^{(N)}\right\|^{2}+c_{n} .
\end{aligned}
$$

It follows that

$$
\begin{aligned}
\left(1-\alpha_{n}-\alpha_{n} \mu \bar{\gamma}\right)\left(1-\beta_{n}-\alpha_{n}-\alpha_{n} \mu \bar{\gamma}\right)\left\|x_{n}-u_{n}^{(N)}\right\|^{2} & \leq\left\|x_{n}-x^{*}\right\|^{2}-\left\|x_{n+1}-x^{*}\right\|^{2}+c_{n} \\
& \leq\left\|x_{n}-x_{n+1}\right\|\left(\left\|x_{n}-x^{*}\right\|+\left\|x_{n+1}-x^{*}\right\|\right)+c_{n} .
\end{aligned}
$$


Therefore, by (3.22) and (3.31), we get

$$
\lim _{n \rightarrow \infty}\left\|x_{n}-u_{n}^{(N)}\right\|=0
$$

Since

$$
\left\|u_{n}^{(N)}-K_{n} W_{n} y_{n}\right\| \leq\left\|u_{n}^{(N)}-x_{n}\right\|+\left\|x_{n}-K_{n} W_{n} y_{n}\right\|
$$

and by (3.26) and (3.70), we have

$$
\lim _{n \rightarrow \infty}\left\|u_{n}^{(N)}-K_{n} W_{n} y_{n}\right\|=0
$$

Since $B$ is a $L_{B}$-Lipschitz continuous and relaxed $(c, d)$-cocoercive mapping on $B$ and $0<\tau<$ $2\left(d-c L_{B}^{2}\right) / L_{B}^{2}$ for any $x^{*} \in \Omega$, we have

$$
\begin{aligned}
\left\|y_{n}-x^{*}\right\|^{2} & =\left\|P_{C}\left(z_{n}-\tau B z_{n}\right)-P_{C}\left(y^{*}-\tau B y^{*}\right)\right\|^{2} \\
& \leq\left\|\left(z_{n}-y^{*}\right)-\tau\left(B z_{n}-B y^{*}\right)\right\|^{2} \\
& =\left\|z_{n}-y^{*}\right\|^{2}-2 \tau\left\langle z_{n}-y^{*}, B z_{n}-B y^{*}\right\rangle+\tau^{2}\left\|B z_{n}-B y^{*}\right\|^{2} \\
& \leq\left\|x_{n}-x^{*}\right\|^{2}-2 \tau\left\{-c\left\|B z_{n}-B y^{*}\right\|^{2}+d\left\|z_{n}-y^{*}\right\|^{2}\right\}+\tau^{2}\left\|B z_{n}-B y^{*}\right\|^{2} \\
& =\left\|x_{n}-x^{*}\right\|^{2}+2 \tau c\left\|B z_{n}-B y^{*}\right\|^{2}-2 \tau d\left\|z_{n}-y^{*}\right\|^{2}+\tau^{2}\left\|B z_{n}-B y^{*}\right\|^{2} \\
& \leq\left\|x_{n}-x^{*}\right\|^{2}+2 \tau c\left\|B z_{n}-B y^{*}\right\|^{2}-\frac{2 \tau d}{L_{B}^{2}}\left\|B z_{n}-B y^{*}\right\|^{2}+\tau^{2}\left\|B y_{n}-B p\right\|^{2} \\
& =\left\|x_{n}-x^{*}\right\|^{2}+\left(2 \tau c+\tau^{2}-\frac{2 \tau d}{L_{B}^{2}}\right)\left\|B z_{n}-B y^{*}\right\|^{2} .
\end{aligned}
$$

Similarly, since $D$ is a $L_{D}$-Lipschitz continuous and relaxed $\left(c^{\prime}, d^{\prime}\right)$-cocoercive mapping on $D$ and $0<\delta<2\left(d^{\prime}-c^{\prime} L_{D}^{2}\right) / L_{D^{\prime}}^{2}$, we also have

$$
\left\|z_{n}-y^{*}\right\|^{2} \leq\left\|x_{n}-x^{*}\right\|^{2}+\left(2 \delta c^{\prime}+\delta^{2}-\frac{2 \delta d^{\prime}}{L_{D}^{2}}\right)\left\|D u_{n}^{(N)}-D x^{*}\right\|^{2} .
$$


Substituting (3.37) into (3.29), we have

$$
\begin{aligned}
\left\|x_{n+1}-x^{*}\right\|^{2} \leq & \left(1-\alpha_{n}-\alpha_{n} \mu \bar{\gamma}\right)\left(1-\beta_{n}-\alpha_{n}-\alpha_{n} \mu \bar{\gamma}\right) \\
& \times\left\{\left\|x_{n}-x^{*}\right\|^{2}+\left(2 \tau c+\tau^{2}-\frac{2 \tau d}{L_{B}^{2}}\right)\left\|B z_{n}-B y^{*}\right\|^{2}\right\} \\
& +\left(1-\alpha_{n}-\alpha_{n} \mu \bar{\gamma}\right) \beta_{n}\left\|x_{n}-x^{*}\right\|^{2}+c_{n} \\
= & \left(1-\alpha_{n}-\alpha_{n} \mu \bar{\gamma}\right)^{2}\left\|x_{n}-x^{*}\right\|^{2} \\
& +\left(1-\alpha_{n}-\alpha_{n} \mu \bar{\gamma}\right)\left(1-\beta_{n}-\alpha_{n}-\alpha_{n} \mu \bar{\gamma}\right)\left(2 \tau c+\tau^{2}-\frac{2 \tau d}{L_{B}^{2}}\right)\left\|B z_{n}-B y^{*}\right\|^{2}+c_{n} \\
\leq & \left\|x_{n}-x^{*}\right\|^{2}+\left(2 \tau c+\tau^{2}-\frac{2 \tau d}{L_{B}^{2}}\right)\left\|B z_{n}-B y^{*}\right\|^{2}+c_{n} .
\end{aligned}
$$

Again, substituting (3.38) into (3.29) and using also (3.10), we get

$$
\begin{aligned}
\left\|x_{n+1}-x^{*}\right\|^{2} \leq & \left(1-\alpha_{n}-\alpha_{n} \mu \bar{\gamma}\right)\left(1-\beta_{n}-\alpha_{n}-\alpha_{n} \mu \bar{\gamma}\right)\left\|y_{n}-x^{*}\right\|^{2} \\
& +\left(1-\alpha_{n}-\alpha_{n} \mu \bar{\gamma}\right) \beta_{n}\left\|x_{n}-x^{*}\right\|^{2}+c_{n} \\
\leq & \left(1-\alpha_{n}-\alpha_{n} \mu \bar{\gamma}\right)\left(1-\beta_{n}-\alpha_{n}-\alpha_{n} \mu \bar{\gamma}\right)\left\|z_{n}-y^{*}\right\|^{2} \\
& +\left(1-\alpha_{n}-\alpha_{n} \mu \bar{\gamma}\right) \beta_{n}\left\|x_{n}-x^{*}\right\|^{2}+c_{n} \\
\leq & \left(1-\alpha_{n}-\alpha_{n} \mu \bar{\gamma}\right)\left(1-\beta_{n}-\alpha_{n}-\alpha_{n} \mu \bar{\gamma}\right) \\
& \times\left\{\left\|x_{n}-x^{*}\right\|^{2}+\left(2 \delta c^{\prime}+\delta^{2}-\frac{2 \delta d^{\prime}}{L_{D}^{2}}\right)\left\|D u_{n}^{(N)}-D x^{*}\right\|^{2}\right\} \\
& +\left(1-\alpha_{n}-\alpha_{n} \mu \bar{\gamma}\right) \beta_{n}\left\|x_{n}-x^{*}\right\|^{2}+c_{n} \\
= & \left(1-\alpha_{n}-\alpha_{n} \mu \bar{\gamma}\right)^{2}\left\|x_{n}-x^{*}\right\|^{2}+\left(1-\alpha_{n}-\alpha_{n} \mu \bar{\gamma}\right)\left(1-\beta_{n}-\alpha_{n}-\alpha_{n} \mu \bar{\gamma}\right) \\
& \times\left(2 \delta c^{\prime}+\delta^{2}-\frac{2 \delta d^{\prime}}{L_{D}^{2}}\right)\left\|D u_{n}^{(N)}-D x^{*}\right\|^{2}+c_{n} \\
\leq & \left\|x_{n}-x^{*}\right\|^{2}+\left(2 \delta c^{\prime}+\delta^{2}-\frac{2 \delta d^{\prime}}{L_{D}^{2}}\right)\left\|D u_{n}^{(N)}-D x^{*}\right\|^{2}+c_{n} .
\end{aligned}
$$

Therefore, by (3.39) and (3.40), we have

$$
\begin{aligned}
\left(\frac{2 \tau d}{L_{B}^{2}}-2 \tau c-\tau^{2}\right)\left\|B z_{n}-B y^{*}\right\|^{2} & \leq\left\|x_{n}-x^{*}\right\|^{2}-\left\|x_{n+1}-x^{*}\right\|^{2}+c_{n} \\
& \leq\left\|x_{n}-x_{n+1}\right\|\left(\left\|x_{n}-x^{*}\right\|+\left\|x_{n+1}-x^{*}\right\|\right)+c_{n} \\
\left(\frac{2 \delta d^{\prime}}{L_{D}^{2}}-2 \delta c^{\prime}-\delta^{2}\right)\left\|D u_{n}^{(N)}-D x^{*}\right\|^{2} & \leq\left\|x_{n}-x^{*}\right\|^{2}-\left\|x_{n+1}-x^{*}\right\|^{2}+c_{n} \\
& \leq\left\|x_{n}-x_{n+1}\right\|\left(\left\|x_{n}-x^{*}\right\|+\left\|x_{n+1}-x^{*}\right\|\right)+c_{n} .
\end{aligned}
$$


It follows from (3.22) and (3.31) that we obtain

$$
\begin{gathered}
\lim _{n \rightarrow \infty}\left\|B z_{n}-B y^{*}\right\|=0, \\
\lim _{n \rightarrow \infty}\left\|D u_{n}^{(N)}-D x^{*}\right\|=0 .
\end{gathered}
$$

From (2.6), we have

$$
\begin{aligned}
\left\|z_{n}-y^{*}\right\|^{2}= & \left\|P_{C}\left(u_{n}^{(N)}-\delta D u_{n}^{(N)}\right)-P_{C}\left(x^{*}-\delta D x^{*}\right)\right\|^{2} \\
\leq & \left\langle\left(u_{n}^{(N)}-\delta D u_{n}^{(N)}\right)-\left(x^{*}-\delta D x^{*}\right), z_{n}-y^{*}\right\rangle \\
= & \frac{1}{2}\left\{\left\|\left(u_{n}^{(N)}-\delta D u_{n}^{(N)}\right)-\left(x^{*}-\delta D x^{*}\right)\right\|^{2}+\left\|z_{n}-y^{*}\right\|^{2}\right. \\
& \left.\quad-\left\|\left[\left(u_{n}^{(N)}-\delta D u_{n}^{(N)}\right)-\left(x^{*}-\delta D x^{*}\right)\right]-\left(z_{n}-y^{*}\right)\right\|^{2}\right\} \\
\leq & \frac{1}{2}\left\{\left\|u_{n}^{(N)}-x^{*}\right\|^{2}+\left\|z_{n}-y^{*}\right\|^{2}-\left\|\left(u_{n}^{(N)}-z_{n}\right)-\left(x^{*}-y^{*}\right)-\delta\left(D u_{n}^{(N)}-D x^{*}\right)\right\|^{2}\right\} \\
= & \frac{1}{2}\left\{\left\|u_{n}^{(N)}-x^{*}\right\|^{2}+\left\|z_{n}-y^{*}\right\|^{2}-\left\|\left(u_{n}^{(N)}-z_{n}\right)-\left(x^{*}-y^{*}\right)\right\|^{2}\right. \\
& \left.\quad+2 \delta\left\langle\left(u_{n}^{(N)}-z_{n}\right)-\left(x^{*}-y^{*}\right), D u_{n}^{(N)}-D x^{*}\right\rangle-\delta^{2}\left\|D u_{n}^{(N)}-D x^{*}\right\|^{2}\right\} \\
\leq & \frac{1}{2}\left\{\left\|x_{n}-x^{*}\right\|^{2}+\left\|z_{n}-y^{*}\right\|^{2}-\left\|\left(u_{n}^{(N)}-z_{n}\right)-\left(x^{*}-y^{*}\right)\right\|^{2}\right. \\
& \left.+2 \delta\left\|\left(u_{n}^{(N)}-z_{n}\right)-\left(x^{*}-y^{*}\right)\right\|\left\|D u_{n}^{(N)}-D x^{*}\right\|-\delta^{2}\left\|D u_{n}^{(N)}-D x^{*}\right\|^{2}\right\} .
\end{aligned}
$$

So, we obtain

$$
\begin{aligned}
\left\|z_{n}-y^{*}\right\|^{2} \leq & \left\|x_{n}-x^{*}\right\|^{2}-\left\|\left(u_{n}^{(N)}-z_{n}\right)-\left(x^{*}-y^{*}\right)\right\|^{2} \\
& +2 \delta\left\|\left(u_{n}^{(N)}-z_{n}\right)-\left(x^{*}-y^{*}\right)\right\|\left\|D u_{n}^{(N)}-D x^{*}\right\|-\delta^{2}\left\|D u_{n}^{(N)}-D x^{*}\right\|^{2} .
\end{aligned}
$$


By (3.29), we get

$$
\begin{aligned}
\left\|x_{n+1}-x^{*}\right\|^{2} \leq & \left(1-\alpha_{n}-\alpha_{n} \mu \bar{\gamma}\right)\left(1-\beta_{n}-\alpha_{n}-\alpha_{n} \mu \bar{\gamma}\right)\left\|y_{n}-x^{*}\right\|^{2} \\
& +\left(1-\alpha_{n}-\alpha_{n} \mu \bar{\gamma}\right) \beta_{n}\left\|x_{n}-x^{*}\right\|^{2}+c_{n} \\
\leq & \left(1-\alpha_{n}-\alpha_{n} \mu \bar{\gamma}\right)\left(1-\beta_{n}-\alpha_{n}-\alpha_{n} \mu \bar{\gamma}\right)\left\|z_{n}-y^{*}\right\|^{2} \\
& +\left(1-\alpha_{n}-\alpha_{n} \mu \bar{\gamma}\right) \beta_{n}\left\|x_{n}-x^{*}\right\|^{2}+c_{n} \\
\leq & \left(1-\alpha_{n}-\alpha_{n} \mu \bar{\gamma}\right)\left(1-\beta_{n}-\alpha_{n}-\alpha_{n} \mu \bar{\gamma}\right) \\
& \times\left\{\left\|x_{n}-x^{*}\right\|^{2}-\left\|\left(u_{n}^{(N)}-z_{n}\right)-\left(x^{*}-y^{*}\right)\right\|^{2}\right. \\
& \left.+2 \delta\left\|\left(u_{n}^{(N)}-z_{n}\right)-\left(x^{*}-y^{*}\right)\right\|\left\|D u_{n}^{(N)}-D x^{*}\right\|-\delta^{2}\left\|D u_{n}^{(N)}-D x^{*}\right\|^{2}\right\} \\
& +\left(1-\alpha_{n}-\alpha_{n} \mu \bar{\gamma}\right) \beta_{n}\left\|x_{n}-x^{*}\right\|^{2}+c_{n} \\
= & \left(1-\alpha_{n}-\alpha_{n} \mu \bar{\gamma}\right)^{2}\left\|x_{n}-x^{*}\right\|^{2}+\left(1-\alpha_{n}-\alpha_{n} \mu \bar{\gamma}\right)\left(1-\beta_{n}-\alpha_{n}-\alpha_{n} \mu \bar{\gamma}\right) \\
& \times\left\{-\left\|\left(u_{n}^{(N)}-z_{n}\right)-\left(x^{*}-y^{*}\right)\right\|^{2}\right. \\
& \left.\quad+2 \delta\left\|\left(u_{n}^{(N)}-z_{n}\right)-\left(x^{*}-y^{*}\right)\right\|\left\|D u_{n}^{(N)}-D x^{*}\right\|-\delta^{2}\left\|D u_{n}^{(N)}-D x^{*}\right\|^{2}\right\}+c_{n} \\
\leq & \left\|x_{n}-x^{*}\right\|^{2}-\left(1-\alpha_{n}-\alpha_{n} \mu \bar{\gamma}\right)\left(1-\beta_{n}-\alpha_{n}-\alpha_{n} \mu \bar{\gamma}\right)\left\|\left(u_{n}^{(N)}-z_{n}\right)-\left(x^{*}-y^{*}\right)\right\|^{2} \\
& +2 \delta\left\|\left(u_{n}^{(N)}-z_{n}\right)-\left(x^{*}-y^{*}\right)\right\|\left\|D u_{n}^{(N)}-D x^{*}\right\| \\
- & \delta^{2}\left(1-\alpha_{n}-\alpha_{n} \mu \bar{\gamma}\right)\left(1-\beta_{n}-\alpha_{n}-\alpha_{n} \mu \bar{\gamma}\right)\left\|D u_{n}^{(N)}-D x^{*}\right\|^{2}+c_{n}
\end{aligned}
$$

which implies that

$$
\begin{aligned}
\left(1-\alpha_{n}\right. & \left.-\alpha_{n} \mu \bar{\gamma}\right)\left(1-\beta_{n}-\alpha_{n}-\alpha_{n} \mu \bar{\gamma}\right)\left\|\left(u_{n}^{(N)}-z_{n}\right)-\left(x^{*}-y^{*}\right)\right\|^{2} \\
\leq & \left\|x_{n}-x^{*}\right\|^{2}-\left\|x_{n+1}-x^{*}\right\|^{2} \\
& +2 \delta\left\|\left(u_{n}^{(N)}-z_{n}\right)-\left(x^{*}-y^{*}\right)\right\|\left\|D u_{n}^{(N)}-D x^{*}\right\| \\
& -\delta^{2}\left(1-\alpha_{n}-\alpha_{n} \mu \bar{\gamma}\right)\left(1-\beta_{n}-\alpha_{n}-\alpha_{n} \mu \bar{\gamma}\right)\left\|D u_{n}^{(N)}-D x^{*}\right\|^{2}+c_{n} \\
\leq & \left\|x_{n}-x_{n+1}\right\|\left(\left\|x_{n}-x^{*}\right\|+\left\|x_{n+1}-x^{*}\right\|\right) \\
& +2 \delta\left\|\left(u_{n}^{(N)}-z_{n}\right)-\left(x^{*}-y^{*}\right)\right\|\left\|D u_{n}^{(N)}-D x^{*}\right\| \\
& -\delta^{2}\left(1-\alpha_{n}-\alpha_{n} \mu \bar{\gamma}\right)\left(1-\beta_{n}-\alpha_{n}-\alpha_{n} \mu \bar{\gamma}\right)\left\|D u_{n}^{(N)}-D x^{*}\right\|^{2}+c_{n} .
\end{aligned}
$$


From (3.22), (3.31), and (3.43), we have

$$
\lim _{n \rightarrow \infty}\left\|\left(u_{n}^{(N)}-z_{n}\right)-\left(x^{*}-y^{*}\right)\right\|=0 .
$$

Now, from (2.2) and (2.7), we observe that

$$
\begin{aligned}
\left\|\left(z_{n}-y_{n}\right)+\left(x^{*}-y^{*}\right)\right\|^{2}= & \|\left(z_{n}-\tau B z_{n}\right)-\left(y^{*}-\tau B y^{*}\right) \\
& -\left[P_{C}\left(z_{n}-\tau B z_{n}\right)-P_{C}\left(y^{*}-\tau B y^{*}\right)\right]+\tau\left(B z_{n}-B y^{*}\right) \|^{2} \\
\leq & \left\|\left(z_{n}-\tau B z_{n}\right)-\left(y^{*}-\tau B y^{*}\right)-\left[P_{C}\left(z_{n}-\tau B z_{n}\right)-P_{C}\left(y^{*}-\tau B y^{*}\right)\right]\right\|^{2} \\
& +2 \tau\left\langle B z_{n}-B y^{*},\left(z_{n}-y_{n}\right)+\left(x^{*}-y^{*}\right)\right\rangle \\
\leq & \left\|\left(z_{n}-\tau B z_{n}\right)-\left(y^{*}-\tau B y^{*}\right)\right\|^{2}-\left\|P_{C}\left(z_{n}-\tau B z_{n}\right)-P_{C}\left(y^{*}-\tau B y^{*}\right)\right\|^{2} \\
& +2 \tau\left\|B z_{n}-B y^{*}\right\|\left\|\left(z_{n}-y_{n}\right)+\left(x^{*}-y^{*}\right)\right\| \\
\leq & \left\|\left(z_{n}-\tau B z_{n}\right)-\left(y^{*}-\tau B y^{*}\right)\right\|^{2} \\
& -\left\|K_{n} W_{n} P_{C}\left(z_{n}-\tau B z_{n}\right)-K_{n} W_{n} P_{C}\left(y^{*}-\tau B y^{*}\right)\right\|^{2} \\
& +2 \tau\left\|B z_{n}-B y^{*}\right\|\left\|\left(z_{n}-y_{n}\right)+\left(x^{*}-y^{*}\right)\right\| \\
= & \left\|\left(z_{n}-\tau B z_{n}\right)-\left(y^{*}-\tau B y^{*}\right)\right\|^{2}-\left\|K_{n} W_{n} y_{n}-K_{n} W_{n} x^{*}\right\|^{2} \\
& +2 \tau\left\|B z_{n}-B y^{*}\right\|\left\|\left(z_{n}-y_{n}\right)+\left(x^{*}-y^{*}\right)\right\| \\
= & \left(\left\|\left(z_{n}-\tau B z_{n}\right)-\left(y^{*}-\tau B y^{*}\right)\right\|-\left\|K_{n} W_{n} y_{n}-x^{*}\right\|\right) \\
& \times\left(\left\|\left(z_{n}-\tau B z_{n}\right)-\left(y^{*}-\tau B y^{*}\right)\right\|+\left\|K_{n} W_{n} y_{n}-x^{*}\right\|\right) \\
& +2 \tau\left\|B z_{n}-B y^{*}\right\|\left\|\left(z_{n}-y_{n}\right)+\left(x^{*}-y^{*}\right)\right\| \\
\leq & \left\|\left(z_{n}-\tau B z_{n}\right)-\left(y^{*}-\tau B y^{*}\right)-\left(K_{n} W_{n} y_{n}-x^{*}\right)\right\| \\
& \times\left(\left\|\left(z_{n}-\tau B z_{n}\right)-\left(y^{*}-\tau B y^{*}\right)\right\|+\left\|K_{n} W_{n} y_{n}-x^{*}\right\|\right) \\
& +2 \tau\left\|B z_{n}-B y^{*}\right\|\left\|\left(z_{n}-y_{n}\right)+\left(x^{*}-y^{*}\right)\right\| \\
= & \left\|\left(u_{n}^{(N)}-K_{n} W_{n} y_{n}\right)+\left(x^{*}-y^{*}\right)-\left(u_{n}^{(N)}-z_{n}\right)-\tau\left(B z_{n}-B y^{*}\right)\right\| \\
& \times\left(\left\|\left(z_{n}-\tau B z_{n}\right)-\left(y^{*}-\tau B y^{*}\right)\right\|+\left\|K_{n} W_{n} y_{n}-x^{*}\right\|\right) \\
& +2 \tau\left\|B z_{n}-B y^{*}\right\|\left\|\left(z_{n}-y_{n}\right)+\left(x^{*}-y^{*}\right)\right\| . \\
&
\end{aligned}
$$

It follows from (3.36), (3.42), and (3.48) that we have

$$
\lim _{n \rightarrow \infty}\left\|\left(z_{n}-y_{n}\right)+\left(x^{*}-y^{*}\right)\right\|=0,
$$

since

$$
\left\|K_{n} W_{n} y_{n}-y_{n}\right\| \leq\left\|K_{n} W_{n} y_{n}-u_{n}^{(N)}\right\|+\left\|\left(u_{n}^{(N)}-z_{n}\right)-\left(x^{*}-y^{*}\right)\right\|+\left\|\left(z_{n}-y_{n}\right)+\left(x^{*}-y^{*}\right)\right\| .
$$


Journal of Applied Mathematics

It follows from (3.36), (3.48) and (3.50), we get

$$
\lim _{n \rightarrow \infty}\left\|K_{n} W_{n} y_{n}-y_{n}\right\|=0
$$

and from (3.26), and (3.52) that we have

$$
\lim _{n \rightarrow \infty}\left\|x_{n}-y_{n}\right\|=0
$$

Since $\left\{W_{n} y_{n}\right\}$ is a bounded sequence in $C$, from Lemma 2.10 for all $s \geq 0$, we have

$$
\begin{aligned}
\lim _{n \rightarrow \infty}\left\|K_{n} W_{n} y_{n}-S(s) K_{n} W_{n} y_{n}\right\| & =\lim _{n \rightarrow \infty}\left\|\frac{1}{t_{n}} \int_{0}^{t_{n}} S(s) W_{n} y_{n} d s-S(s)\left(\frac{1}{t_{n}} \int_{0}^{t_{n}} S(s) W_{n} y_{n} d s\right)\right\| \\
& =0,
\end{aligned}
$$

and since

$$
\begin{aligned}
\left\|y_{n}-S(s) y_{n}\right\| & \leq\left\|y_{n}-K_{n} W_{n} y_{n}\right\|+\left\|K_{n} W_{n} y_{n}-S(s) K_{n} W_{n} y_{n}\right\|+\left\|S(s) K_{n} W_{n} y_{n}-S(s) y_{n}\right\| \\
& \leq 2\left\|y_{n}-K_{n} W_{n} y_{n}\right\|+\left\|K_{n} W_{n} y_{n}-S(s) K_{n} W_{n} y_{n}\right\|,
\end{aligned}
$$

it follows from (3.52) and (3.54) that we get

$$
\lim _{n \rightarrow \infty}\left\|y_{n}-S(s) y_{n}\right\|=0
$$

On the other hand, since $J_{r_{k}}^{\Theta_{k}}: H \rightarrow H$ is firmly nonexpansive, $\mathcal{A}^{k}:=J_{r_{k}}^{\Theta_{k}} \cdots$ $J_{r_{2}}^{\Theta_{2}} J_{r_{1}}^{\Theta_{1}}, k=1,2, \ldots, N$ and $x^{*} \in \Omega$, we have

$$
\begin{aligned}
\left\|\mathscr{A}^{k+1} x_{n}-x^{*}\right\|^{2} & =\left\|J_{r_{k+1}}^{\Theta_{k+1}} \mathcal{A}^{k} x_{n}-J_{r_{k+1}}^{\Theta_{k+1}} x^{*}\right\|^{2} \\
& \leq\left\langle J_{r_{k+1}}^{\Theta_{k+1}} \mathscr{A}^{k} x_{n}-x^{*}, \mathcal{A}^{k} x_{n}-x^{*}\right\rangle \\
& =\frac{1}{2}\left(\left\|J_{r_{k+1}}^{\Theta_{k+1}} \mathcal{A}^{k} x_{n}-x^{*}\right\|^{2}+\left\|\mathcal{A}^{k} x_{n}-x^{*}\right\|^{2}-\left\|J_{r_{k+1}}^{\Theta_{k+1}} \mathcal{A}^{k} x_{n}-\mathcal{A}^{k} x_{n}\right\|^{2}\right),
\end{aligned}
$$

and hence

$$
\left\|\mathscr{A}^{k+1} x_{n}-x^{*}\right\|^{2} \leq\left\|x_{n}-x^{*}\right\|^{2}-\left\|\mathscr{A}^{k+1} x_{n}-\mathscr{A}^{k} x_{n}\right\|^{2} .
$$


From (3.10), (3.29), and (3.58), for each $k=1,2, \ldots, N-1$, we have

$$
\begin{aligned}
\left\|x_{n+1}-x^{*}\right\|^{2} \leq & \left(1-\alpha_{n}-\alpha_{n} \mu \bar{\gamma}\right)\left(1-\beta_{n}-\alpha_{n}-\alpha_{n} \mu \bar{\gamma}\right)\left\|y_{n}-x^{*}\right\|^{2} \\
& +\left(1-\alpha_{n}-\alpha_{n} \mu \bar{\gamma}\right) \beta_{n}\left\|x_{n}-x^{*}\right\|^{2}+c_{n} \\
\leq & \left(1-\alpha_{n}-\alpha_{n} \mu \bar{\gamma}\right)\left(1-\beta_{n}-\alpha_{n}-\alpha_{n} \mu \bar{\gamma}\right)\left\|\mathcal{A}^{k} x_{n}-x^{*}\right\|^{2} \\
& +\left(1-\alpha_{n}-\alpha_{n} \mu \bar{\gamma}\right) \beta_{n}\left\|x_{n}-x^{*}\right\|^{2}+c_{n} \\
\leq & \left(1-\alpha_{n}-\alpha_{n} \mu \bar{\gamma}\right)\left(1-\beta_{n}-\alpha_{n}-\alpha_{n} \mu \bar{\gamma}\right)\left\|\mathcal{A}^{k+1} x_{n}-x^{*}\right\|^{2} \\
& +\left(1-\alpha_{n}-\alpha_{n} \mu \bar{\gamma}\right) \beta_{n}\left\|x_{n}-x^{*}\right\|^{2}+c_{n} \\
\leq & \left(1-\alpha_{n}-\alpha_{n} \mu \bar{\gamma}\right)\left(1-\beta_{n}-\alpha_{n}-\alpha_{n} \mu \bar{\gamma}\right)\left\{\left\|x_{n}-x^{*}\right\|^{2}-\left\|\mathcal{A}^{k+1} x_{n}-\mathcal{A}^{k} x_{n}\right\|^{2}\right\} \\
& +\left(1-\alpha_{n}-\alpha_{n} \mu \bar{\gamma}\right) \beta_{n}\left\|x_{n}-x^{*}\right\|^{2}+c_{n} \\
= & \left(1-\alpha_{n}-\alpha_{n} \mu \bar{\gamma}\right)^{2}\left\|x_{n}-x^{*}\right\|^{2} \\
& -\left(1-\alpha_{n}-\alpha_{n} \mu \bar{\gamma}\right)\left(1-\beta_{n}-\alpha_{n}-\alpha_{n} \mu \bar{\gamma}\right)\left\|\mathcal{A}^{k+1} x_{n}-\mathcal{A}^{k} x_{n}\right\|^{2}+c_{n} \\
\leq & \left\|x_{n}-x^{*}\right\|^{2} \\
& -\left(1-\alpha_{n}-\alpha_{n} \mu \bar{\gamma}\right)\left(1-\beta_{n}-\alpha_{n}-\alpha_{n} \mu \bar{\gamma}\right)\left\|\mathcal{A}^{k+1} x_{n}-\mathcal{A}^{k} x_{n}\right\|^{2}+c_{n} .
\end{aligned}
$$

It follows that

$$
\begin{aligned}
& \left(1-\alpha_{n}-\alpha_{n} \mu \bar{\gamma}\right)\left(1-\beta_{n}-\alpha_{n}-\alpha_{n} \mu \bar{\gamma}\right)\left\|\mathcal{A}^{k+1} x_{n}-\mathcal{A}^{k} x_{n}\right\|^{2} \\
& \quad \leq\left\|x_{n}-x^{*}\right\|^{2}-\left\|x_{n+1}-x^{*}\right\|^{2}+c_{n} \\
& \quad \leq\left\|x_{n}-x_{n+1}\right\|\left(\left\|x_{n}-x^{*}\right\|+\left\|x_{n+1}-x^{*}\right\|\right)+c_{n} .
\end{aligned}
$$

Therefore, by (3.22) and (3.31), we get

$$
\lim _{n \rightarrow \infty}\left\|\mathscr{A}^{k+1} x_{n}-\mathscr{A}^{k} x_{n}\right\|=0 \text { that is } \lim _{n \rightarrow \infty}\left\|u_{n}^{(k+1)}-u_{n}^{(k)}\right\|=0 \text {. }
$$

Step 4 . We prove that

$$
\limsup _{n \rightarrow \infty}\left\langle u+[\gamma f-(I+\mu A)] x^{*}, x_{n}-x^{*}\right\rangle \leq 0,
$$

where $x^{*}$ is a solution of the optimization problem:

$$
\min _{x \in \Omega} \frac{\mu}{2}\left\langle A x^{*}, x^{*}\right\rangle+\frac{1}{2}\left\|x^{*}-u\right\|^{2}-h\left(x^{*}\right)
$$


To show this inequality, we can choose a subsequence $\left\{y_{n_{i}}\right\}$ of $\left\{y_{n}\right\}$ such that

$$
\lim _{i \rightarrow \infty}\left\langle u+[\gamma f-(I+\mu A)] x^{*}, y_{n_{i}}-x^{*}\right\rangle=\limsup _{n \rightarrow \infty}\left\langle u+[\gamma f-(I+\mu A)] x^{*}, y_{n}-x^{*}\right\rangle .
$$

Since $\left\{y_{n_{i}}\right\}$ is bounded, there exists a subsequence $\left\{y_{n_{i_{j}}}\right\}$ of $\left\{y_{n_{i}}\right\}$ which converges weakly to $z \in C$. Without loss of generality, we can assume that $y_{n_{i}} \rightarrow z$. From (3.53), we get $x_{n_{i}} \rightarrow z$.

Next, we show that $z \in \Omega:=F(\mathcal{S}) \cap F(W) \cap \mathfrak{F} \cap \operatorname{SVI}(C, B, D)$, where $\mathfrak{F}=$ $\left(\cap_{k=1}^{N} \operatorname{MEP}\left(\Theta_{k}, \phi\right)\right)$.

(1) First, we prove that $z \in F(\mathcal{S})$. Indeed, from Lemma 2.11 and (3.56), we get $z \in$ $F(S)$, that is, $z=S(s) z$, for all $s \geq 0$.

(2) Next, we show that $z \in F(W)=\cap_{n=1}^{\infty} F\left(W_{n}\right)$, where $F\left(W_{n}\right)=\cap_{i=1}^{n} F\left(T_{i}\right)$, for all $n \geq 1$ and $F\left(W_{n+1}\right) \subset F\left(W_{n}\right)$. Assume that $z \notin F(W)$, then there exists a positive integer $m$ such that $z \notin F\left(T_{m}\right)$ and so $z \notin \cap_{i=1}^{m} F\left(T_{i}\right)$. Hence for any $n \geq m, z \notin \cap_{i=1}^{n} F\left(T_{i}\right)=F\left(W_{n}\right)$, that is, $z \neq W_{n} z$. This together with $z=S(s) z$, for all $s \geq 0$, shows $z=S(s) z \neq S(s) W_{n} z$, for all $s \geq 0$; therefore, we have $z \neq K_{n} W_{n} z$, for all $n \geq m$. It follows from the Opial's condition and (3.52) that

$$
\begin{aligned}
\liminf _{i \rightarrow \infty}\left\|y_{n_{i}}-z\right\| & <\liminf _{i \rightarrow \infty}\left\|y_{n_{i}}-K_{n_{i}} W_{n_{i}} z\right\| \\
& \leq \liminf _{i \rightarrow \infty}\left(\left\|y_{n_{i}}-K_{n_{i}} W_{n_{i}} y_{n_{i}}\right\|+\left\|K_{n_{i}} W_{n_{i}} y_{n_{i}}-K_{n_{i}} W_{n_{i}} z\right\|\right) \\
& \leq \liminf _{i \rightarrow \infty}\left\|y_{n_{i}}-z\right\|,
\end{aligned}
$$

which is a contradiction. Thus, we get $z \in F(W)$.

(3) Now, we prove that $z \in \mathfrak{F}$. Since $\mathcal{A}^{k+1}=J_{r_{k+1}}^{\Theta_{k+1}} \mathscr{A}^{k}, k=1,2, \ldots, N-1$, and $u_{n}^{(k+1)}=$ $A^{k+1} x_{n}$, we have

$$
\Theta\left(\mathscr{A}^{k+1} x_{n}, x\right)+\phi(x)-\phi\left(\mathscr{A}^{k+1} x_{n}\right)+\frac{1}{r_{k+1}}\left\langle K^{\prime}\left(\mathcal{A}^{k+1} x_{n}\right)-K^{\prime}\left(\mathcal{A}^{k} x_{n}\right), \eta\left(x, \mathcal{A}^{k+1} x_{n}\right)\right\rangle \geq 0,
$$

It follows that

$$
\frac{1}{r_{k+1}}\left\langle K^{\prime}\left(\mathcal{A}^{k+1} x_{n_{i}}\right)-K^{\prime}\left(\mathcal{A}^{k} x_{n_{i}}\right), \eta\left(x, \mathcal{A}^{k+1} x_{n_{i}}\right)\right\rangle \geq-\Theta\left(\mathcal{A}^{k+1} x_{n_{i}}, x\right)-\phi(x)+\phi\left(\mathscr{A}^{k+1} x_{n_{i}}\right)
$$

for all $x \in H$. From (3.61) and by conditions (C1)(c) and (C2), we get

$$
\lim _{n_{i} \rightarrow \infty} \frac{1}{r_{k+1}}\left\langle K^{\prime}\left(\mathcal{A}^{k+1} x_{n_{i}}\right)-K^{\prime}\left(\mathcal{A}^{k} x_{n_{i}}\right), \eta\left(x, \mathcal{A}^{k+1} x_{n_{i}}\right)\right\rangle=0 .
$$

By the assumption that $\phi$ is lower semicontinuous, then it is weakly lower semicontinuous and by the condition $(\mathrm{H} 2)$ that $x \mapsto\left(-\Theta_{i}(x, y)\right)$ is lower semicontinuous, then it is weakly 
lower semicontinuous. Since $y_{n_{i}} \rightarrow z$, it follows from (3.36), (3.52), and (3.61) that $u_{n_{i}}^{(k)} \rightarrow z$ for each $k=1,2, \ldots, N-1$. Taking the lower limit $n_{i} \rightarrow \infty$ in (3.67), we have

$$
\Theta_{k+1}(z, x)+\phi(x)-\phi(z) \geq 0, \quad \forall x \in H, \forall k=0,1,2, \ldots, N-1
$$

Therefore, $z \in \cap_{k=1}^{N} \operatorname{MEP}\left(\Theta_{k}, \phi\right)$.

(4) Next, we show that $z \in \operatorname{SVI}(C, B, D)$. By (3.36) and (3.52), we have

$$
\left\|u_{n}^{(N)}-y_{n}\right\| \leq\left\|u_{n}^{(N)}-K_{n} W_{n} y_{n}\right\|+\left\|K_{n} W_{n} y_{n}-y_{n}\right\| \longrightarrow 0 \quad \text { as } n \longrightarrow \infty .
$$

By Lemma 2.13 that $G$ is a nonexpansive, we obtain

$$
\begin{aligned}
\left\|y_{n}-G\left(y_{n}\right)\right\| & =\left\|P_{C}\left[P_{C}\left(u_{n}^{(N)}-\delta D u_{n}^{(N)}\right)-\tau B P_{C}\left(u_{n}^{(N)}-\delta D u_{n}^{(N)}\right)\right]-G\left(y_{n}\right)\right\| \\
& =\left\|G\left(u_{n}^{(N)}\right)-G\left(y_{n}\right)\right\| \\
& \leq\left\|u_{n}^{(N)}-y_{n}\right\| .
\end{aligned}
$$

Thus,

$$
\lim _{n \rightarrow \infty}\left\|y_{n}-G\left(y_{n}\right)\right\|=0
$$

By Lemma 2.14, we obtain that $z \in \operatorname{SVI}(C, B, D)$. Hence $z \in \Omega$ is proved.

Now, from Lemma 2.9, (3.64), and (3.53), we have

$$
\begin{aligned}
\limsup _{n \rightarrow \infty}\left\langle u+[\gamma f-(I+\mu A)] x^{*}, x_{n}-x^{*}\right\rangle & =\limsup _{n \rightarrow \infty}\left\langle u+[\gamma f-(I+\mu A)] x^{*}, y_{n}-x^{*}\right\rangle \\
& =\lim _{i \rightarrow \infty}\left\langle u+[\gamma f-(I+\mu A)] x^{*}, y_{n_{i}}-x^{*}\right\rangle \\
& =\left\langle u+[\gamma f-(I+\mu A)] x^{*}, z-x^{*}\right\rangle \leq 0 .
\end{aligned}
$$

By (3.52), (3.53), and (3.73), we obtain

$$
\limsup _{n \rightarrow \infty}\left\langle u+[\gamma f-(I+\mu A)] x^{*}, K_{n} W_{n} y_{n}-x^{*}\right\rangle \leq 0
$$


Step 5. Finally, we show that $x_{n} \rightarrow x^{*}$. From (3.1), we obtain

$$
\begin{aligned}
& \left\|x_{n+1}-x^{*}\right\|^{2} \\
& =\left\|\alpha_{n}\left(u+\gamma f\left(W_{n} x_{n}\right)\right)+\beta_{n} x_{n}+\left(\left(1-\beta_{n}\right) I-\alpha_{n}(I+\mu A)\right) K_{n} W_{n} y_{n}-x^{*}\right\|^{2} \\
& =\|\left(\left(1-\beta_{n}\right) I-\alpha_{n}(I+\mu A)\right)\left(K_{n} W_{n} y_{n}-x^{*}\right)+\beta_{n}\left(x_{n}-x^{*}\right) \\
& +\alpha_{n}\left(u+\gamma f\left(W_{n} x_{n}\right)-(I+\mu A) x^{*}\right) \|^{2} \\
& =\left\|\left(\left(1-\beta_{n}\right) I-\alpha_{n}(I+\mu A)\right)\left(K_{n} W_{n} y_{n}-x^{*}\right)+\beta_{n}\left(x_{n}-x^{*}\right)\right\|^{2} \\
& +2 \alpha_{n}\left\langle\left(\left(1-\beta_{n}\right) I-\alpha_{n}(I+\mu A)\right)\left(K_{n} W_{n} y_{n}-x^{*}\right), u+\gamma f\left(W_{n} x_{n}\right)-(I+\mu A) x^{*}\right\rangle \\
& +2 \alpha_{n} \beta_{n}\left\langle x_{n}-x^{*}, u+\gamma f\left(W_{n} x_{n}\right)-(I+\mu A) x^{*}\right\rangle+\alpha_{n}^{2}\left\|u+\gamma f\left(W_{n} x_{n}\right)-(I+\mu A) x^{*}\right\|^{2} \\
& \leq\left[\left(1-\beta_{n}-\alpha_{n}(1+\mu \bar{\gamma})\right)\left\|K_{n} W_{n} y_{n}-x^{*}\right\|+\beta_{n}\left\|x_{n}-x^{*}\right\|\right]^{2} \\
& +2 \alpha_{n}\left(1-\beta_{n}\right) \gamma\left\langle K_{n} W_{n} y_{n}-x^{*}, f\left(W_{n} x_{n}\right)-f\left(x^{*}\right)\right\rangle \\
& +2 \alpha_{n}\left(1-\beta_{n}\right)\left\langle K_{n} W_{n} y_{n}-x^{*}, u+\gamma f\left(x^{*}\right)-(I+\mu A) x^{*}\right\rangle \\
& -2 \alpha_{n}^{2} \gamma\left\langle(I+\mu A)\left(K_{n} W_{n} y_{n}-x^{*}\right), f\left(W_{n} x_{n}\right)-f\left(x^{*}\right)\right\rangle \\
& -2 \alpha_{n}^{2}\left\langle(I+\mu A)\left(K_{n} W_{n} y_{n}-x^{*}\right), u+\gamma f\left(x^{*}\right)-(I+\mu A) x^{*}\right\rangle \\
& +2 \alpha_{n} \beta_{n} \gamma\left\langle x_{n}-x^{*}, f\left(W_{n} x_{n}\right)-f\left(x^{*}\right)\right\rangle+2 \alpha_{n} \beta_{n}\left\langle x_{n}-x^{*}, u+\gamma f\left(x^{*}\right)-(I+\mu A) x^{*}\right\rangle \\
& +\alpha_{n}^{2}\left\|u+\gamma f\left(W_{n} x_{n}\right)-(I+\mu A) x^{*}\right\|^{2} \\
& \leq\left[\left(1-\beta_{n}-\alpha_{n}(1+\mu \bar{\gamma})\right)\left\|K_{n} W_{n} y_{n}-x^{*}\right\|+\beta_{n}\left\|x_{n}-x^{*}\right\|\right]^{2} \\
& +2 \alpha_{n}\left(1-\beta_{n}\right) \gamma\left\|K_{n} W_{n} y_{n}-x^{*}\right\|\left\|f\left(W_{n} x_{n}\right)-f\left(x^{*}\right)\right\| \\
& +2 \alpha_{n}\left(1-\beta_{n}\right)\left\langle K_{n} W_{n} y_{n}-x^{*}, u+\gamma f\left(x^{*}\right)-(I+\mu A) x^{*}\right\rangle \\
& -2 \alpha_{n}^{2} \gamma\left\|(I+\mu A)\left(K_{n} W_{n} y_{n}-x^{*}\right)\right\|\left\|f\left(W_{n} x_{n}\right)-f\left(x^{*}\right)\right\| \\
& -2 \alpha_{n}^{2}\left\|(I+\mu A)\left(K_{n} W_{n} y_{n}-x^{*}\right)\right\|\left\|u+\gamma f\left(x^{*}\right)-(I+\mu A) x^{*}\right\| \\
& +2 \alpha_{n} \beta_{n} \gamma\left\|x_{n}-x^{*}\right\|\left\|f\left(W_{n} x_{n}\right)-f\left(x^{*}\right)\right\|+2 \alpha_{n} \beta_{n}\left\langle x_{n}-x^{*}, u+\gamma f\left(x^{*}\right)-(I+\mu A) x^{*}\right\rangle \\
& +\alpha_{n}^{2}\left\|u+\gamma f\left(W_{n} x_{n}\right)-(I+\mu A) x^{*}\right\|^{2} \\
& \leq\left[\left(1-\beta_{n}-\alpha_{n}(1+\mu \bar{\gamma})\right)\left\|x_{n}-x^{*}\right\|+\beta_{n}\left\|x_{n}-x^{*}\right\|\right]^{2} \\
& +2 \alpha_{n}\left(1-\beta_{n}\right) \gamma \alpha\left\|x_{n}-x^{*}\right\|^{2} \\
& +2 \alpha_{n}\left(1-\beta_{n}\right)\left\langle K_{n} W_{n} y_{n}-x^{*}, u+\gamma f\left(x^{*}\right)-(I+\mu A) x^{*}\right\rangle \\
& \text { - } 2 \alpha_{n}^{2} \gamma \alpha\left\|(I+\mu A)\left(K_{n} W_{n} y_{n}-x^{*}\right)\right\|\left\|x_{n}-x^{*}\right\| \\
& -2 \alpha_{n}^{2}\left\|(I+\mu A)\left(K_{n} W_{n} y_{n}-x^{*}\right)\right\|\left\|u+\gamma f\left(x^{*}\right)-(I+\mu A) x^{*}\right\| \\
& +2 \alpha_{n} \beta_{n} \gamma \alpha\left\|x_{n}-x^{*}\right\|^{2}+2 \alpha_{n} \beta_{n}\left\langle x_{n}-x^{*}, u+\gamma f\left(x^{*}\right)-(I+\mu A) x^{*}\right\rangle
\end{aligned}
$$




$$
\begin{aligned}
& +\alpha_{n}^{2}\left\|u+\gamma f\left(W_{n} x_{n}\right)-(I+\mu A) x^{*}\right\|^{2} \\
& =\left[\left(1-\alpha_{n}(1+\mu \bar{\gamma})\right)+2 \alpha_{n} \gamma \alpha\right]\left\|x_{n}-x^{*}\right\|^{2} \\
& +\alpha_{n}\left\{2\left(1-\beta_{n}\right)\left\langle K_{n} W_{n} y_{n}-x^{*}, u+\gamma f\left(x^{*}\right)-(I+\mu A) x^{*}\right\rangle\right. \\
& -2 \alpha_{n} \gamma \alpha\left\|(I+\mu A)\left(K_{n} W_{n} y_{n}-x^{*}\right)\right\|\left\|x_{n}-x^{*}\right\| \\
& -2 \alpha_{n}\left\|(I+\mu A)\left(K_{n} W_{n} y_{n}-x^{*}\right)\right\|\left\|u+\gamma f\left(x^{*}\right)-(I+\mu A) x^{*}\right\| \\
& +2 \beta_{n}\left\langle x_{n}-x^{*}, u+\gamma f\left(x^{*}\right)-(I+\mu A) x^{*}\right\rangle \\
& \left.+\alpha_{n}\left\|u+\gamma f\left(W_{n} x_{n}\right)-(I+\mu A) x^{*}\right\|^{2}\right\} \\
& =\left[1-2 \alpha_{n}(1+\mu \bar{\gamma})+\alpha_{n}^{2}(1+\mu \bar{\gamma})^{2}+2 \alpha_{n} \gamma \alpha\right]\left\|x_{n}-x^{*}\right\|^{2} \\
& +\alpha_{n}\left\{2\left(1-\beta_{n}\right)\left\langle K_{n} W_{n} y_{n}-x^{*}, u+\gamma f\left(x^{*}\right)-(I+\mu A) x^{*}\right\rangle\right. \\
& -2 \alpha_{n} \gamma \alpha\left\|(I+\mu A)\left(K_{n} W_{n} y_{n}-x^{*}\right)\right\|\left\|x_{n}-x^{*}\right\| \\
& -2 \alpha_{n}\left\|(I+\mu A)\left(K_{n} W_{n} y_{n}-x^{*}\right)\right\|\left\|u+\gamma f\left(x^{*}\right)-(I+\mu A) x^{*}\right\| \\
& +2 \beta_{n}\left\langle x_{n}-x^{*}, u+\gamma f\left(x^{*}\right)-(I+\mu A) x^{*}\right\rangle \\
& \left.+\alpha_{n}\left\|u+\gamma f\left(W_{n} x_{n}\right)-(I+\mu A) x^{*}\right\|^{2}\right\} . \\
& =\left[1-2 \alpha_{n}(1+\mu \bar{\gamma}-\gamma \alpha)\right]\left\|x_{n}-x^{*}\right\|^{2} \\
& +\alpha_{n}\left\{2\left(1-\beta_{n}\right)\left\langle K_{n} W_{n} y_{n}-x^{*}, u+\gamma f\left(x^{*}\right)-(I+\mu A) x^{*}\right\rangle\right. \\
& +2 \beta_{n}\left\langle x_{n}-x^{*}, u+\gamma f\left(x^{*}\right)-(I+\mu A) x^{*}\right\rangle \\
& +\alpha_{n}\left[(1+\mu \bar{\gamma})^{2}\left\|x_{n}-x^{*}\right\|^{2}\right. \\
& -2 \gamma \alpha\left\|(I+\mu A)\left(K_{n} W_{n} y_{n}-x^{*}\right)\right\|\left\|x_{n}-x^{*}\right\| \\
& -2\left\|(I+\mu A)\left(K_{n} W_{n} y_{n}-x^{*}\right)\right\|\left\|u+\gamma f\left(x^{*}\right)-(I+\mu A) x^{*}\right\| \\
& \left.\left.+\left\|u+\gamma f\left(W_{n} x_{n}\right)-(I+\mu A) x^{*}\right\|^{2}\right]\right\} \text {. }
\end{aligned}
$$

Since $\left\{x_{n}\right\},\left\{f\left(W_{n} x_{n}\right)\right\}$, and $\left\{K_{n} W_{n} y_{n}\right\}$ are bounded, there exist $M>0$ such that

$$
\begin{aligned}
(1+\mu \bar{\gamma})^{2}\left\|x_{n}-x^{*}\right\|^{2} \\
\quad-2 \gamma \alpha\left\|(I+\mu A)\left(K_{n} W_{n} y_{n}-x^{*}\right)\right\|\left\|x_{n}-x^{*}\right\| \\
\quad-2\left\|(I+\mu A)\left(K_{n} W_{n} y_{n}-x^{*}\right)\right\|\left\|u+\gamma f\left(x^{*}\right)-(I+\mu A) x^{*}\right\| \\
\quad+\left\|u+\gamma f\left(W_{n} x_{n}\right)-(I+\mu A) x^{*}\right\|^{2} \leq M
\end{aligned}
$$


for all $n \geq 0$. It follows that

$$
\left\|x_{n+1}-x^{*}\right\|^{2} \leq\left(1-\alpha_{n} a_{n}\right)\left\|x_{n}-x^{*}\right\|^{2}+\alpha_{n} b_{n}
$$

where

$$
\begin{aligned}
a_{n}= & 2(1+\mu \bar{\gamma}-\gamma \alpha), \\
b_{n}= & 2\left(1-\beta_{n}\right)\left\langle K_{n} W_{n} y_{n}-x^{*}, u+\gamma f\left(x^{*}\right)-(I+\mu A) x^{*}\right\rangle \\
& +2 \beta_{n}\left\langle x_{n}-x^{*}, u+\gamma f\left(x^{*}\right)-(I+\mu A) x^{*}\right\rangle+\alpha_{n} M .
\end{aligned}
$$

Applying Lemma 2.8 to (3.77), we conclude that $x_{n} \rightarrow x^{*}$. This completes the proof.

Remark 3.2. For example, of the control conditions (C4)-(C6), we set $\alpha_{n}=1 / 10 n, \beta_{n}=n /(n+$ 1 ). We set $B, D$ is a 1 -Lipschitz continuous and relaxed $(0,1)$-cocoercive mapping, (i.e., $L_{B}=$ $1=L_{D}$ and $\left.c=0=c^{\prime}, d=1=d^{\prime}\right)$.

Then, we can choose $\tau \in(0,2)$ and $\delta \in(0,2)$ which satisfies the condition (C6) in Theorem 3.1.

Corollary 3.3. Let $C$ be a nonempty closed convex subset of a real Hilbert space $H$ which $C+C \subset C$ and let $f$ be a contraction of $C$ into itself with $\alpha \in(0,1)$. Let $\phi$ be a lower semicontinuous and convex functional from $H$ to $\mathbb{R}$ and let $\Theta: H \times H \rightarrow \mathbb{R}$ be a finite family of equilibrium functions satisfying conditions (H1)-(H3). Let $\mathcal{S}=\{S(s): 0 \leq s<\infty\}$ be a nonexpansive semigroup on $C$ and let $\left\{t_{n}\right\}$ be a positive real divergent sequence. Let $\left\{V_{i}: C \rightarrow C\right\}_{i=1}^{\infty}$ be a countable family of uniformly $k$ - strict pseudo-contractions, let $\left\{T_{i}: C \rightarrow C\right\}_{i=1}^{\infty}$ be the countable family of nonexpansive mappings defined by $T_{i} x=t x+(1-t) V_{i} x$, for all $x \in C$, for all $i \geq 1, t \in[k, 1)$, let $W_{n}$ be the $W$-mapping defined by (2.12), and let $W$ be a mapping defined by (2.13) with $F(W) \neq \emptyset$. Let $A$ be a strongly positive linear bounded operator on $H$ with coefficient $\bar{\gamma}>0$ and let $0<\gamma<(1+\mu \bar{\gamma}) / \alpha, B: H \rightarrow H$ be a $L_{B}$-Lipschitz continuous and relaxed $(c, d)$-cocoercive mapping with $d>c L_{B}^{2}$, and let $D: H \rightarrow H$ be a $L_{D}$-Lipschitz continuous and relaxed $\left(c^{\prime}, d^{\prime}\right)$-cocoercive mapping with $d^{\prime}>c^{\prime} L_{D}^{2}$. Suppose that $\Omega:=F(\mathcal{S}) \cap F(W) \cap \operatorname{MEP}(\Theta, \mathrm{E}) \cap \operatorname{SVI}(\mathrm{C}, \mathrm{B}, \mathrm{D}) \neq \emptyset$. Let $\mu>0, \gamma>0$ and $r>0$, which are constants. For given $x_{1} \in H$ arbitrarily and fixed $u \in H$, suppose $\left\{x_{n}\right\},\left\{y_{n}\right\},\left\{z_{n}\right\}$, and $\left\{u_{n}\right\}$ are the sequences generated iteratively by

$$
\begin{gathered}
\Theta\left(u_{n}, x\right)+\phi(x)-\phi\left(u_{n}\right)+\frac{1}{r}\left\langle K^{\prime}\left(u_{n}\right)-K^{\prime}\left(x_{n}\right), \eta\left(x, u_{n}\right)\right\rangle \geq 0, \quad \forall x \in H, \\
z_{n}=P_{C}\left(u_{n}-\delta D u_{n}\right), \\
y_{n}=P_{C}\left(z_{n}-\tau B z_{n}\right),
\end{gathered}
$$

where $u_{n}=J_{r}^{\Theta} x_{n}$ such that $J_{r}^{\Theta}: H \rightarrow H$ is the mapping defined by (2.22) and $\left\{\alpha_{n}\right\}$ and $\left\{\beta_{n}\right\}$ are two sequences in $(0,1)$ for all $n \in \mathbb{N}$. If the functions $\eta: H \times H \rightarrow H$ and $K: H \rightarrow \mathbb{R}$ satisfy the 
conditions (C1)-(C6) as given in Theorem 3.1, then $\left\{x_{n}\right\}$ converges strongly to $x^{*} \in \Omega$, which solves the following optimization problem $(O P)$ :

$$
\min _{x^{*} \in \Omega} \frac{\mu}{2}\left\langle A x^{*}, x^{*}\right\rangle+\frac{1}{2}\left\|x^{*}-u\right\|^{2}-h\left(x^{*}\right)
$$

and $\left(x^{*}, y^{*}\right)$ is a solution of the general system of variational inequality problem (1.20) such that $y^{*}=P_{C}\left(x^{*}-\delta D x^{*}\right)$.

Proof. Taking $N=1$ in Theorem 3.1. Hence, the conclusion follows. This completes the proof.

Corollary 3.4. Let $C$ be a nonempty closed convex subset of a real Hilbert space $H$ which $C+C \subset C$ and let $f$ be a contraction of $C$ into itself with $\alpha \in(0,1)$. Let $\mathcal{S}=\{S(s): 0 \leq s<\infty\}$ be a nonexpansive semigroup on $C$ and let $\left\{t_{n}\right\}$ be a positive real divergent sequence. Let $\left\{V_{i}: C \rightarrow C\right\}_{i=1}^{\infty}$ be a countable family of uniformly $k$-strict pseudo-contractions, let $\left\{T_{i}: C \rightarrow C\right\}_{i=1}^{\infty}$ be the countable family of nonexpansive mappings defined by $T_{i} x=t x+(1-t) V_{i} x$, for all $x \in C$, for all $i \geq 1, t \in$ $[k, 1)$, let $W_{n}$ be the $W$-mapping defined by (2.12), and let $W$ be a mapping defined by (2.13) with $F(W) \neq \emptyset$. Let $A$ be a strongly positive linear bounded operator on $H$ with coefficient $\bar{\gamma}>0$ and let $0<\gamma<(1+\mu \bar{\gamma}) / \alpha, B: H \rightarrow H$ be a $L_{B}$-Lipschitz continuous and relaxed $(c, d)$-cocoercive mapping with $d>c L_{B^{\prime}}^{2}$ and let $D: H \rightarrow H$ be a $L_{D}$-Lipschitz continuous and relaxed $\left(c^{\prime}, d^{\prime}\right)$ cocoercive mapping with $d^{\prime}>c^{\prime} L_{D}^{2}$. Suppose that $\Omega:=F(S) \cap F(W) \cap \operatorname{SVI}(\mathrm{C}, \mathrm{B}, \mathrm{D}) \neq \emptyset$. Let $\mu>0$ and $\gamma>0$, which are constants. For given $x_{1} \in H$ arbitrarily and fixed $u \in H$, suppose $\left\{x_{n}\right\},\left\{y_{n}\right\}$, and $\left\{z_{n}\right\}$ are the sequences generated iteratively by

$$
\begin{gathered}
z_{n}=P_{C}\left(x_{n}-\delta D x_{n}\right), \\
y_{n}=P_{C}\left(z_{n}-\tau B z_{n}\right), \\
x_{n+1}=\alpha_{n}\left[u+\gamma f\left(W_{n} x_{n}\right)\right]+\beta_{n} x_{n}+\left[\left(1-\beta_{n}\right) I-\alpha_{n}(I+\mu A)\right] \frac{1}{t_{n}} \int_{0}^{t_{n}} S(s) W_{n} y_{n} d s,
\end{gathered}
$$

where $\left\{\alpha_{n}\right\}$ and $\left\{\beta_{n}\right\}$ are two sequences in $(0,1)$ for all $n \in \mathbb{N}$. If the sequence $\left\{x_{n}\right\}$ satisfy the conditions (C1)-(C6) as given in Theorem 3.1, then $\left\{x_{n}\right\}$ converges strongly to $x^{*} \in \Omega$, which solves the following optimization problem $(O P)$ :

$$
\min _{x^{*} \in \Omega} \frac{\mu}{2}\left\langle A x^{*}, x^{*}\right\rangle+\frac{1}{2}\left\|x^{*}-u\right\|^{2}-h\left(x^{*}\right)
$$

and $\left(x^{*}, y^{*}\right)$ is a solution of the general system of variational inequality problem (1.20) such that $y^{*}=P_{C}\left(x^{*}-\delta D x^{*}\right)$.

Proof. Put $\Theta(x, y) \equiv \phi(x) \equiv 0$ for all $x, y \in H$ and $r=1$. Take $K(x)=\|x\|^{2} / 2$ and $\eta(y, x)=$ $y-x$, for all $x, y \in H$. Then, we get $u_{n}=P_{C} x_{n}=x_{n}$ in Corollary 3.3. Hence, the conclusion follows. This completes the proof.

Corollary 3.5. Let $C$ be a nonempty closed convex subset of a real Hilbert space $H$ and let $f$ be a contraction of $H$ into itself with $\alpha \in(0,1)$. Let $S=\{S(s): 0 \leq s<\infty\}$ be a nonexpansive semigroup on $C$ and let $\left\{t_{n}\right\}$ be a positive real divergent sequence. Let $A$ be a strongly positive linear bounded 
operator on $H$ with coefficient $\bar{\gamma}>0$ and let $0<\gamma<(1+\mu \bar{\gamma}) / \alpha, B: H \rightarrow H$ be a $L_{B}$-Lipschitz continuous and relaxed $(c, d)$-cocoercive mapping with $d>c L_{B}^{2}$. Suppose that $\Omega:=F(S) \cap B^{-1} 0 \neq \emptyset$. Let $\mu>0$ and $\gamma>0$, which are constants. For given $x_{1} \in H$ arbitrarily and fixed $u \in H$, suppose the $\left\{x_{n}\right\},\left\{y_{n}\right\}$, and $\left\{z_{n}\right\}$ are the sequences generated iteratively by

$$
\begin{gathered}
z_{n}=x_{n}-\tau B x_{n}, \\
y_{n}=z_{n}-\tau B z_{n} \\
x_{n+1}=\alpha_{n}\left[u+\gamma f\left(x_{n}\right)\right]+\beta_{n} x_{n}+\left[\left(1-\beta_{n}\right) I-\alpha_{n}(I+\mu A)\right] \frac{1}{t_{n}} \int_{0}^{t_{n}} S(s) y_{n} d s,
\end{gathered}
$$

where $\left\{\alpha_{n}\right\}$ and $\left\{\beta_{n}\right\}$ are two sequences in $(0,1)$ for all $n \in \mathbb{N}$. If the sequence $\left\{x_{n}\right\}$ satisfy the conditions (C1)-(C6) as given in Theorem 3.1, then $\left\{x_{n}\right\}$ converges strongly to $x^{*} \in \Omega$.

Proof. Setting $\tau=\delta, C \equiv H, D \equiv B$ and $W_{n} \equiv P_{H} \equiv I$ in Corollary 3.4, it follows from the proof of Theorem 4.1 in [25] that $B^{-1} 0=\operatorname{VI}(H, B)$. Hence, the conclusion follows. This completes the proof.

\section{Acknowledgments}

The authors would like to thank the "Centre of Excellence in Mathematics" under the Commission on Higher Education, Ministry of Education, Thailand. Moreover, the authors are grateful to the reviewers for the careful reading of the paper and for the suggestions which improved the quality of this work.

\section{References}

[1] R. U. Verma, "Generalized system for relaxed cocoercive variational inequalities and projection methods," Journal of Optimization Theory and Applications, vol. 121, no. 1, pp. 203-210, 2004.

[2] R. U. Verma, "General convergence analysis for two-step projection methods and applications to variational problems," Applied Mathematics Letters, vol. 18, no. 11, pp. 1286-1292, 2005.

[3] S. Saeidi, "Modified hybrid steepest-descent methods for variational inequalities and fixed points," Mathematical and Computer Modelling, vol. 52, no. 1-2, pp. 134-142, 2010.

[4] P. L. Combettes, "Hilbertian convex feasibility problem: convergence of projection methods," Applied Mathematics and Optimization, vol. 35, no. 3, pp. 311-330, 1997.

[5] F. Deutsch and I. Yamada, "Minimizing certain convex functions over the intersection of the fixed point sets of nonexpansive mappings," Numerical Functional Analysis and Optimization, vol. 19, no. 1-2, pp. 33-56, 1998.

[6] H. K. Xu, "An iterative approach to quadratic optimization," Journal of Optimization Theory and Applications, vol. 116, no. 3, pp. 659-678, 2003.

[7] I. Yamada, N. Ogura, Y. Yamashita, and K. Sakaniwa, "Quadratic optimization of fixed points of nonexpansive mappings in Hilbert space," Numerical Functional Analysis and Optimization, vol. 19, no. 1-2, pp. 165-190, 1998.

[8] A. S. Antipin, F. P. Vasilev, and A. S. Stukalov, "The regularized Newton method for solving equilibrium programming problems with an inexactly specified set," Computational Mathematics and Mathematical Physics, vol. 47, no. 1, pp. 21-33, 2007.

[9] F. Cianciaruso, G. Marino, and L. Muglia, "Iterative methods for equilibrium and fixed point problems for nonexpansive semigroups in Hilbert spaces," Journal of Optimization Theory and Applications, vol. 146, no. 2, pp. 491-509, 2010.

[10] S. D. Flam and A. S. Antipin, "Equilibrium programming using proximal-like algorithms," Mathematical Programming, vol. 78, no. 1, pp. 29-41, 1997. 
[11] A. Moudafi and M. Thera, "Proximal and dynamical approaches to equilibrium problems," in IllPosed Variational Problems and Regularization Techniques (Trier, 1998), vol. 477 of Lecture note in Economics and Mathematical Systems, pp. 187-201, Springer, Berlin, Germany, 1999.

[12] A. S. Stukalov, "An extraproximal method for solving equilibrium problems in a Hilbert space," Computational Mathematics and Mathematical Physics, vol. 46, no. 5, pp. 743-761, 2006.

[13] S. Takahashi and W. Takahashi, "Viscosity approximation methods for equilibrium problems and fixed point problems in Hilbert spaces," Journal of Mathematical Analysis and Applications, vol. 331, no. 1, pp. 506-515, 2007.

[14] P. Sunthrayuth and P. Kumam, "A general iterative algorithm for the solution of variational inequalities for a nonexpansive semigroup in Banach spaces," Journal of Nonlinear Analysis and Optimization, vol. 1, no. 1, pp. 139-150, 2010.

[15] P. Kumam and K. Wattanawitoon, "A general composite explicit iterative scheme of fixed point solutions of variational inequalities for nonexpansive semigroups," Mathematical and Computer Modelling, vol. 53, no. 5-6, pp. 998-1006, 2011.

[16] P. Kumam, U. Hamphries, and P. Katchang, “Common solutions of generalized mixed equilibrium problems, variational inclusions, and common fixed points for nonexpansive semigroups and strictly pseudocontractive mappings," Journal of Applied Mathematics, Article ID 953903, 28 pages, 2011.

[17] T. Jitpeera and P. Kumam, "A new hybrid algorithm for a system of mixed equilibrium problems, fixed point problems for nonexpansive semigroup, and variational inclusion problem," Fixed Point Theory and Applications, Article ID 217407, 27 pages, 2011.

[18] P. Sunthrayuth and P. Kumam, "A new general iterative method for solution of a new general system of variational inclusions for nonexpansive semigroups in Banach spaces," Journal of Applied Mathematics, Article ID 187052, 29 pages, 2011.

[19] P. Kumam and C. Jaiboon, "A system of generalized mixed equilibrium problems and fixed point problems for pseudocontractive mappings in Hilbert spaces," Fixed Point Theory and Applications, Article ID 361512, 33 pages, 2010.

[20] P. Kumam and C. Jaiboon, "Approximation of common solutions to system of mixed equilibrium problems, variational inequality problem, and strict pseudo-contractive mappings," Fixed Point Theory and Applications, Article ID 347204, 30 pages, 2011.

[21] T. Jitpeera and P. Kumam, "A new hybrid algorithm for a system of equilibrium problems and variational inclusion," Annali dell'Universitá di Ferrara, vol. 57, no. 1, pp. 89-108, 2011.

[22] P. Sunthrayuth and P. Kumam, "A system of generalized mixed equilibrium problems, maximal monotone operators and fixed point problems with application to optimization problems," Abstract and Applied Analysis, vol. 2012, Article ID 316276, 39 pages, 2012.

[23] P. Sunthrayuth and P. Kumam, "An iterative method for solving a system of mixed equilibrium problems, system of quasi variational inclusions and fixed point problems of nonexpansive semigroups with application to optimization problems," Abstract and Applied Analysis, vol. 2012, Article ID 979870, 30 pages, 2012.

[24] S.-S. Chang, C. K. Chan, H. W. J. Lee, and L. Yang, "A system of mixed equilibrium problems, fixed point problems of strictly pseudo-contractive mappings and nonexpansive semi-groups," Applied Mathematics and Computation, vol. 216, no. 1, pp. 51-60, 2010.

[25] L.-C. Ceng, C.-y. Wang, and J.-C. Yao, "Strong convergence theorems by a relaxed extragradient method for a general system of variational inequalities," Mathematical Methods of Operations Research, vol. 67, no. 3, pp. 375-390, 2008.

[26] R. U. Verma, "On a new system of nonlinear variational inequalities and associated iterative algorithms," Mathematical Sciences Research Hot-Line, vol. 3, no. 8, pp. 65-68, 1999.

[27] R. U. Verma, "Iterative algorithms and a new system of nonlinear quasivariational inequalities," Advances in Nonlinear Variational Inequalities, vol. 4, no. 1, pp. 117-124, 2001.

[28] F. Cianciaruso, G. Marino, L. Muglia, and Y. Yao, "A hybrid projection algorithm for finding solutions of mixed equilibrium problem and variational inequality problem," Fixed Point Theory and Applications, Article ID 383740, 19 pages, 2010.

[29] L. C. Zeng, S. Schaible, and J. C. Yao, "Iterative algorithm for generalized set-valued strongly nonlinear mixed variational-like inequalities," Journal of Optimization Theory and Applications, vol. 124, no. 3, pp. 725-738, 2005.

[30] J.-C. Yao and O. Chadli, "Pseudomonotone complementarity problems and variational inequalities," in Handbook of Generalized Convexity and Generalized Monotonicity, J. P. Crouzeix, N. Haddjissas, and S. Schaible, Eds., vol. 76, pp. 501-558, Kluwer Academic, Boston, Mass, USA, 2005. 
[31] W. Kumam, P. Junlouchai, and P. Kumam, "Generalized systems of variational inequalities and projection methods for inverse-strongly monotone mappings," Discrete Dynamics in Nature and Society, Article ID 976505, 23 pages, 2011.

[32] R. Wangkeeree and U. Kamraksa, "An iterative approximation method for solving a general system of variational inequality problems and mixed equilibrium problems," Nonlinear Analysis. Hybrid Systems, vol. 3, no. 4, pp. 615-630, 2009.

[33] C. Jaiboon and P. Kumam, "A general iterative method for addressing mixed equilibrium problems and optimization problems," Nonlinear Analysis. Theory, Methods E Applications, vol. 73, no. 5, pp. 1180-1202, 2010.

[34] P. Kumam and C. Jaiboon, "A new hybrid iterative method for mixed equilibrium problems and variational inequality problem for relaxed cocoercive mappings with application to optimization problems," Nonlinear Analysis. Hybrid Systems, vol. 3, no. 4, pp. 510-530, 2009.

[35] H. Zhou, "Convergence theorems of fixed points for k-strict pseudo-contractions in Hilbert spaces," Nonlinear Analysis. Theory, Methods E Applications, vol. 69, no. 2, pp. 456-462, 2008.

[36] K. Shimoji and W. Takahashi, "Strong convergence to common fixed points of infinite nonexpansive mappings and applications," Taiwanese Journal of Mathematics, vol. 5, no. 2, pp. 387-404, 2001.

[37] S. S. Chang, Variational Inequalities and Related Problems, Chongqing Publishing House, Changjiang, China, 2007.

[38] Z. Opial, "Weak convergence of the sequence of successive approximations for nonexpansive mappings," Bulletin of the American Mathematical Society, vol. 73, pp. 591-597, 1967.

[39] G. Marino and H.-K. Xu, "A general iterative method for nonexpansive mappings in Hilbert spaces," Journal of Mathematical Analysis and Applications, vol. 318, no. 1, pp. 43-52, 2006.

[40] M. A. Hanson, "On sufficiency of the Kuhn-Tucker conditions," Journal of Mathematical Analysis and Applications, vol. 80, no. 2, pp. 545-550, 1981.

[41] Q. H. Ansari and J. C. Yao, “Iterative schemes for solving mixed variational-like inequalities," Journal of Optimization Theory and Applications, vol. 108, no. 3, pp. 527-541, 2001.

[42] L.-C. Ceng and J.-C. Yao, "A hybrid iterative scheme for mixed equilibrium problems and fixed point problems," Journal of Computational and Applied Mathematics, vol. 214, no. 1, pp. 186-201, 2008.

[43] T. Suzuki, "Strong convergence of Krasnoselskii and Mann's type sequences for one-parameter nonexpansive semigroups without Bochner integrals," Journal of Mathematical Analysis and Applications, vol. 305, no. 1, pp. 227-239, 2005.

[44] H.-K. Xu, "Viscosity approximation methods for nonexpansive mappings," Journal of Mathematical Analysis and Applications, vol. 298, no. 1, pp. 279-291, 2004.

[45] Y. Yao, M. A. Noor, S. Zainab, and Y.-C. Liou, "Mixed equilibrium problems and optimization problems," Journal of Mathematical Analysis and Applications, vol. 354, no. 1, pp. 319-329, 2009.

[46] T. Shimizu and W. Takahashi, "Strong convergence to common fixed points of families of nonexpansive mappings," Journal of Mathematical Analysis and Applications, vol. 211, no. 1, pp. 71-83, 1997.

[47] K.-K. Tan and H. K. Xu, “The nonlinear ergodic theorem for asymptotically nonexpansive mappings in Banach spaces," Proceedings of the American Mathematical Society, vol. 114, no. 2, pp. 399-404, 1992.

[48] K. Goebel and W. A. Kirk, Topics in Metric Fixed Point Theory, vol. 28, Cambridge University Press, Cambridge, UK, 1990. 


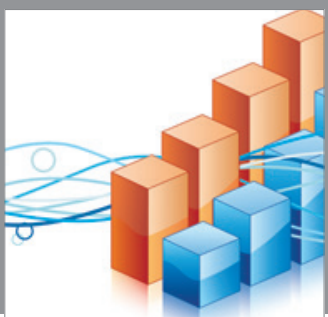

Advances in

Operations Research

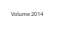

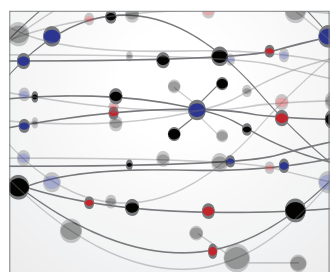

\section{The Scientific} World Journal
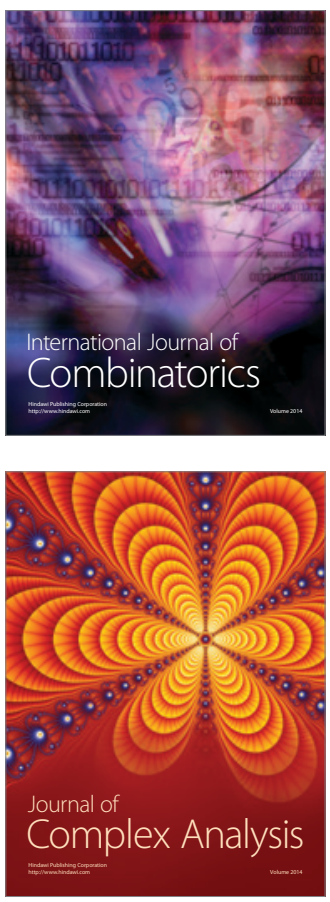

International Journal of

Mathematics and

Mathematical

Sciences
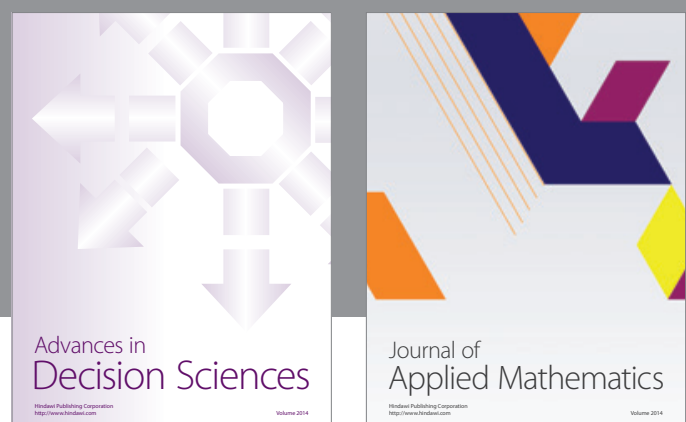

Journal of

Applied Mathematics
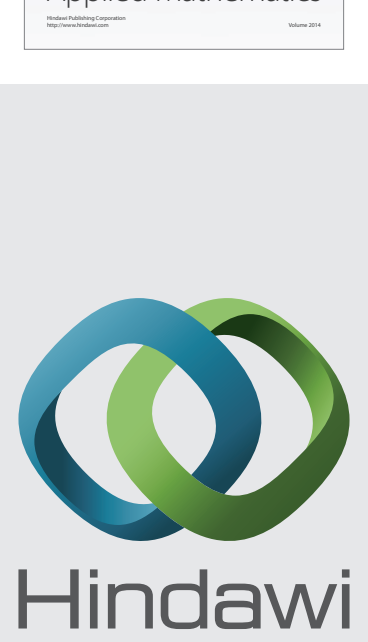

Submit your manuscripts at http://www.hindawi.com
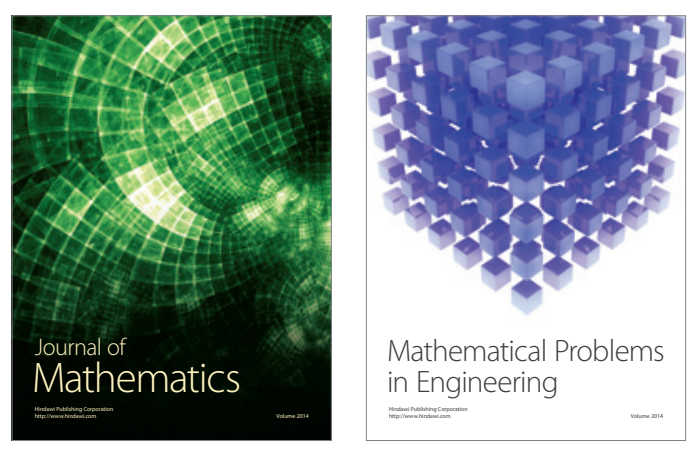

Mathematical Problems in Engineering
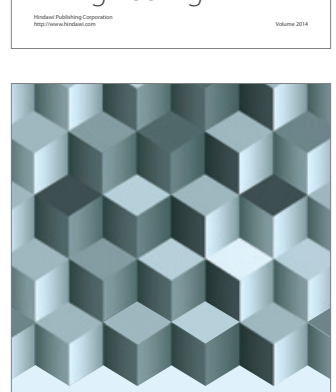

Journal of

Function Spaces
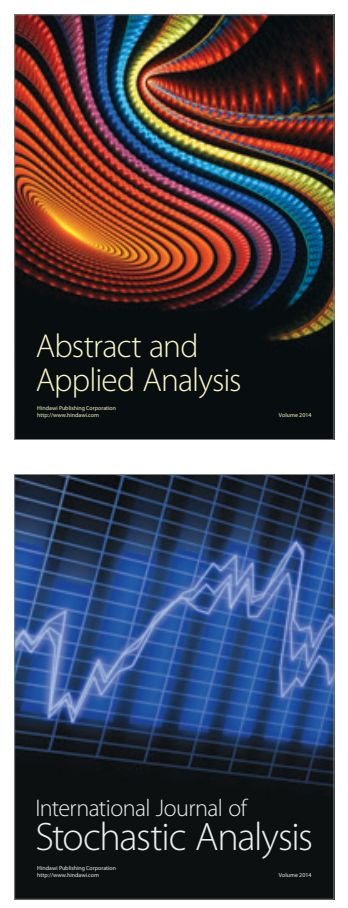

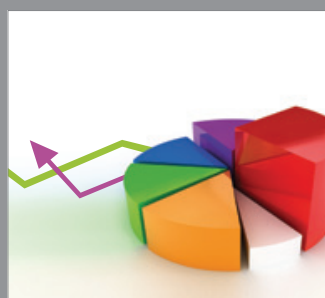

ournal of

Probability and Statistics

Promensencen
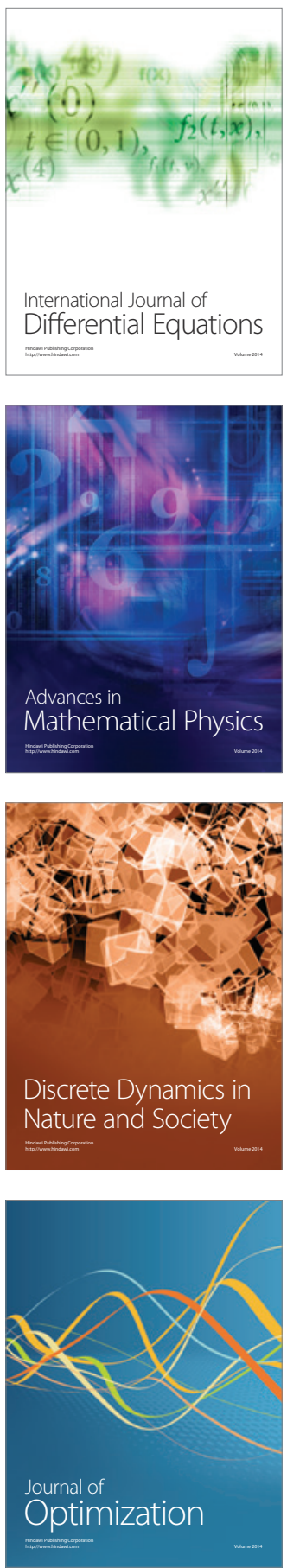